\title{
Effect of Droplet Transition on the Dynamic Behavior of the Keyhole during 6061 Aluminum Alloy Laser-MIG Hybrid Welding
}

\section{Yue Li}

Nanjing University of Aeronautics and Astronautics

\section{Yanqiu Zhao}

Nanjing University of Aeronautics and Astronautics

\section{Xudong Zhou}

Nanjing University of Aeronautics and Astronautics

Xiaohong Zhan ( $\square$ xiaohongzhan_nuaa@126.com )

Nanjing University of Aeronautics and Astronautics

\section{Research Article}

Keywords: Droplet, Keyhole, Thermal-fluid coupling simulation, Laser-MIG hybrid welding

Posted Date: May 11th, 2021

DOI: https://doi.org/10.21203/rs.3.rs-486469/v1

License: (9) This work is licensed under a Creative Commons Attribution 4.0 International License. Read Full License

Version of Record: A version of this preprint was published at The International Journal of Advanced Manufacturing Technology on November 11th, 2021. See the published version at https://doi.org/10.1007/s00170-021-08270-1. 


\title{
Effect of droplet transition on the dynamic behavior of the keyhole during 6061 aluminum alloy laser-MIG hybrid welding
}

Yue Li ${ }^{\text {a }}$, Yanqiu Zhao a Xudong Zhou ${ }^{\text {a }}$, Xiaohong Zhan ${ }^{\mathrm{a}}$

a College of Materials Science and Technology, Nanjing University of Aeronautics and Astronautics, Nanjing 211106, China

Corresponding author: Xiaohong Zhan, xiaohongzhan_nuaa@126.com

\begin{abstract}
The simulation method in laser-MIG hybrid welding, which involves two heat sources and multiple welding parameters, is beneficial to reveal the complex physical phenomena and dynamic behavior of molten pool keyhole during welding process. In this investigation, laser-MIG hybrid welding for 6-mm-thick 6061 aluminum alloy was performed under different heat input by the high-power disc laser, MIG welding system and KUKA Robot. The high-speed camera system was used to observe the droplet transition phenomenon in the welding process. Besides, a thermal-fluid coupling model was established to simulate the temperature field and flow field, which were changed by the droplet transfer during laser-MIG hybrid welding. The experimental and simulated results showed that the droplet transition behavior affected the formation of the keyhole. The keyhole was the smallest when the droplet contacted the molten pool. In addition, the droplet transition brought external momentum and energy to the molten pool, which was conducive to the increase of the flow rate of the molten pool.
\end{abstract}

Keywords Droplet $\cdot$ Keyhole $\cdot$ Thermal-fluid coupling simulation $\cdot$ Laser-MIG hybrid welding

\section{Introduction}

Contemporary aerospace is based on the goal of improving the carrying capacity through weight reduction[1,2]. Aluminum alloys have been widely used in the aerospace field owing to their small density, high specific strength and corrosion resistance[3, 4]. At present, there are numerous problems in the traditional arc welding, such as low welding efficiency, large heat input and large deformation after welding, which seriously limits its application[5]. Although aluminum alloy has high reflectivity to laser, a keyhole is still generated at a high energy density, i.e. greater than $10^{6}$ $\mathrm{W} / \mathrm{cm}^{2}$, which greatly improves the laser absorption rate of aluminum alloy[6, 7]. Laser welding for aluminum alloy is gradually used in aerospace, vehicle, rail transportation and other fields due to its 
advantages of obvious weight loss, slight deformation and high welding efficiency[8, 9]. However, laser welding exists the weaknesses of poor bridging ability and strict requirement of the joint gap[10]. As an applicative and emerging deep penetration connection technology, laser-arc hybrid welding technology, which developed in the 1990s, has been widely applied in civil passenger aircraft, automobiles, construction machinery, rail transportation and equipment manufacturing fields[11, 12]. In the laser-arc hybrid welding process, the dual heat sources synergy and interact effect between laser and arc produces narrow and deep welds, thereby greatly improving production efficiency[13]. Laser-arc hybrid welding with the characteristics of high welding energy, strong gap bridging ability and high joint quality[14], makes up for the deficiencies in the single processing heat source, meanwhile, effectively combines the advantages of the two welding methods[15].

Many researches have been carried out to observe the morphology of the molten pool during the laser-MIG hybrid welding process by high-speed cameras. Faraji et al. [16] used laser-MIG hybrid to fabricate 3-mm-thick 2198 aluminum alloy, and the mechanical property of the welded joint was compared and analyzed. It was found that comparing with a single laser heat source, the keyhole with the deep penetration was easy to obtained even at higher welding speed. Moreover, the micro-hardness and microstructure of high laser power with low arc power were better. Liu et al. [17] observed the droplet transfer and the keyhole in the molten pool during laser-arc hybrid welding by high speed camera. It revealed that the laser had a compressive effect on the arc pressure with the surface of the molten pool. Zhang et al. [18] performed laser-MAG hybrid welding of large thickness steel and investigated the influence of the ratio of laser power to arc power on the weld morphology. What is more, the image of the molten droplet and arc morphology were observed with the help of a high-speed camera system. It was revealed that as the ratio of laser to arc energy increases, the aspect ratio of the weld exhibits a linear increase.

In recent years, the research method of simulation was used to explain complex physical phenomena, which promoted the research on the mechanism level. Lu et al. [19] proposed a three-dimensional transient model coupled with fluid flow, bubble motion and solidification to study the process of keyhole-induced porosity. The simulation results showed that the number of porosity was mainly determined by the frequency of keyhole collapse. As the laser power increased, as well as the welding speed and spot diameter decreased, the keyhole tended to collapse more easily. Guo et al. [20] performed laser-GMAW hybrid welding of $6 \mathrm{~mm}$ aluminum alloy, and simulated the 
formation of keyhole-induced pores with the help of two-dimensional flow field calculations. It was found that under the action of the arc force, relatively stable counterclockwise vortices appeared in the middle and rear of the composite molten pool, which tended to prevent the bubbles from moving to the low temperature area at the rear of the molten pool, thus helping the bubbles to float upward.

However, the research on the droplet transfer behavior in the laser-MIG hybrid welding process is still insufficient. It is not enough to study on the effect of droplet transition on the temperature field distribution of the molten pool. In this paper, with the help of high-speed camera and the establishment of a thermal-fluid coupling model, the droplet transition behavior is studied.

\section{Experimental details}

\subsection{Experimental material and setup}

The base metal used in this paper is 6061-T6 aluminum alloy, its chemical composition is shown in Table 1. The size of aluminum alloy plate is $150 \mathrm{~mm} \times 30 \mathrm{~mm} \times 6 \mathrm{~mm}$, with a Y-shaped groove, as shown in Fig. 2(a). The selected filler wire is ER4047 with a diameter of $1.2 \mathrm{~mm}$ whose main chemical composition is shown in Table 1. The laser-MIG hybrid welding experiment equipment is adopted TruDisk-12003 disc laser with a maximum laser output of $12000 \mathrm{~W}$, KR60HA robot, TPS 5000 Fronius welding machine and self-designed installation fixture. Besides, CP70 high-speed camera is performed to take pictures of the molten pool morphology and droplet transition during the welding process. The experimental equipment is shown in Fig. 1.

Table 1 Chemical composition of 6061 aluminum alloy and ER4047 (wt.\%)

\begin{tabular}{cccccccccc}
\hline Composition & $\mathrm{Si}$ & $\mathrm{Fe}$ & $\mathrm{Cu}$ & $\mathrm{Mn}$ & $\mathrm{Mg}$ & $\mathrm{Zn}$ & $\mathrm{Ti}$ & $\mathrm{Cr}$ & $\mathrm{Al}$ \\
\hline 6061 & 0.56 & 0.70 & 0.30 & 0.89 & 0.93 & 0.25 & 0.15 & 0.04 & Balance \\
ER4047 & 12 & 0.8 & 0.03 & 0.15 & 0.1 & 0.2 & - & - & Balance \\
\hline
\end{tabular}




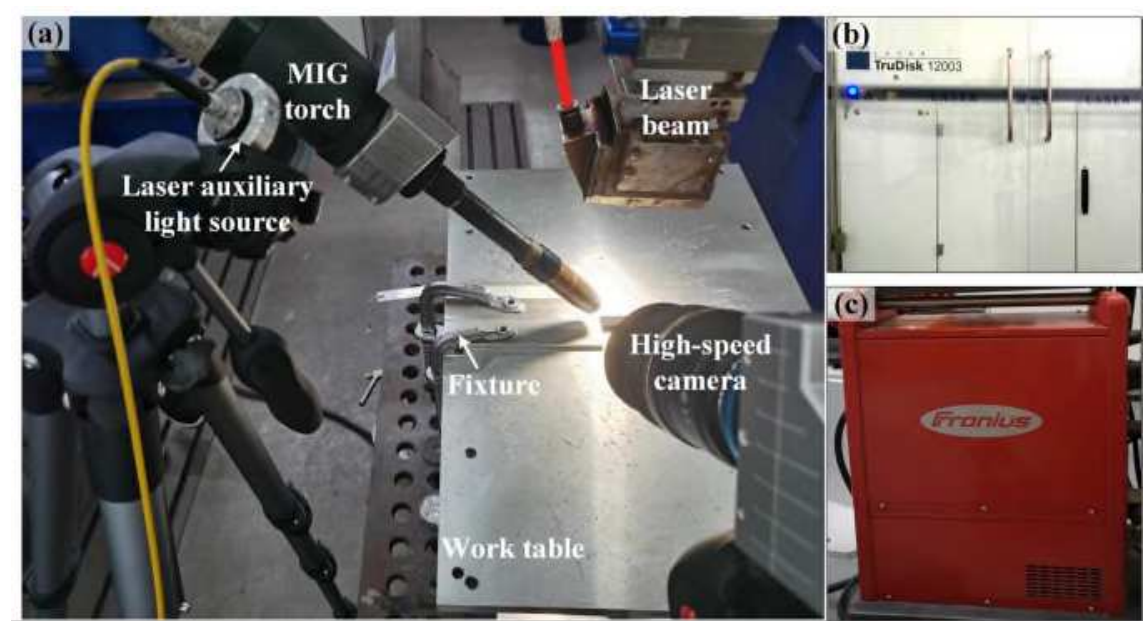

Fig. 1 Experimental setup of 6061 aluminum alloy laser-MIG hybrid welding. (a) Equipment and work table. (b)

Disc laser. (c) Fronius welder

\subsection{Experimental process}

During the laser-MIG hybrid welding of 6061 aluminum alloy, the aluminum alloy plate is connected to the negative electrode effectively, considering the dense oxide film on the surface of aluminum alloy. The cathode crushing effect in arc welding is instrumental to remove the oxide film and facilitate the droplet transition. The schematic diagram of laser-MIG hybrid welding is as shown in Fig. 2. In order to prevent the damage of laser head by the vertical laser irradiation, the angle between the laser beam and the workpiece is $86.5^{\circ}$. At the same time, the angle between the MIG welding torch and the aluminum alloy plate is $45^{\circ}$ considering the interference of the experimental equipment. Argon with purity of $99 \%$ is used as welding shielding gas and its flow rate is $15 \mathrm{~L} / \mathrm{min}$. The welding parameters adopted in this experiment is as shown in Table 2.
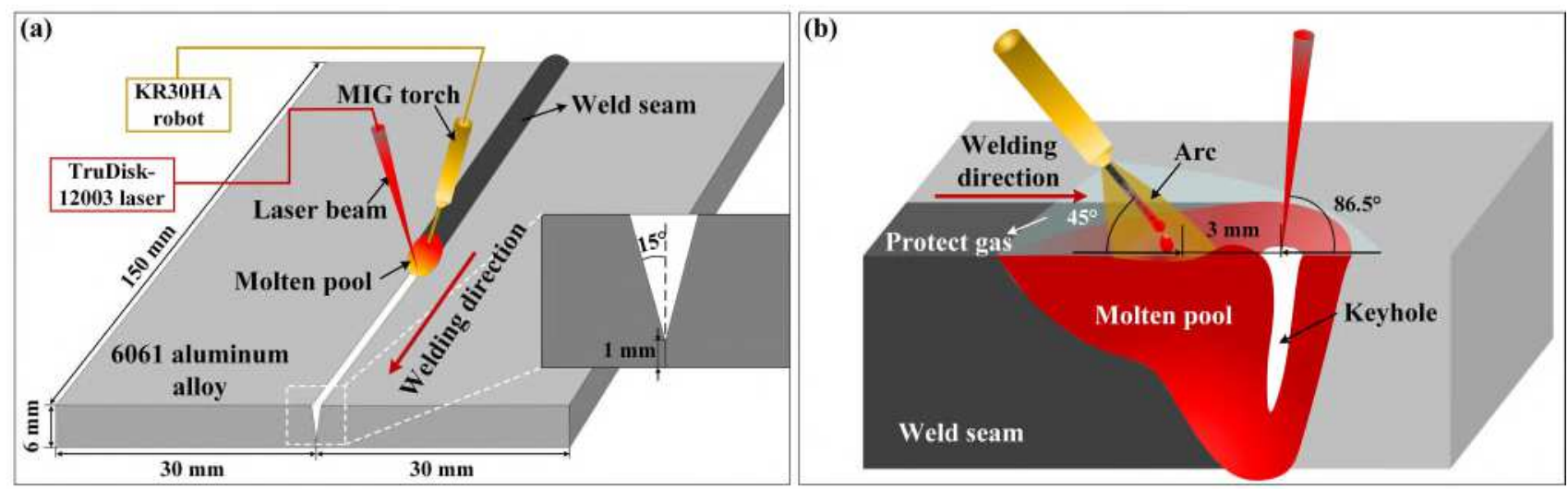

Fig. 2 Schematic diagram of laser-MIG hybrid welding. (a) Welding. (b)Molten pool morphology 
Table 2 The welding parameters in laser-MIG hybrid welding process

\begin{tabular}{ccccc}
\hline Case & Laser power $(\mathrm{kW})$ & Arc current $(\mathrm{A})$ & Welding speed $(\mathrm{m} / \mathrm{min})$ & Filling speed $(\mathrm{m} / \mathrm{min})$ \\
\hline 1 & 4.0 & 90 & 1.2 & 5.3 \\
2 & 4.0 & 100 & 1.2 & 5.7 \\
3 & 4.5 & 120 & 1.2 & 7.0 \\
\hline
\end{tabular}

\section{Mathematical modeling}

\subsection{Governing equations}

The flow of the molten pool and the behavior of metal vapor during the laser-MIG hybrid welding process are both complex physical phenomena[21]. It is followed the law of conservation of mass, energy and momentum, which govern the thermodynamics and kinetics of the molten pool owing to the fluid characteristics at the welding process[22]. The formulas of the three laws are as follows.

Continuity equation

$$
\frac{\partial \rho}{\partial t}+\frac{\partial(\rho u)}{\partial x}+\frac{\partial(\rho v)}{\partial y}+\frac{\partial(\rho w)}{\partial z}=0
$$

especially for incompressible fluids, $\frac{\partial \rho}{\partial t}=0$.

Energy equation

$$
\rho\left[\frac{\partial H}{\partial t}+\left(u-u_{0}\right) \frac{\partial H}{\partial x}+v \frac{\partial H}{\partial y}+w \frac{\partial H}{\partial z}\right]=\frac{\partial}{\partial x}\left(k \frac{\partial T}{\partial x}\right)+\frac{\partial}{\partial y}\left(k \frac{\partial T}{\partial y}\right)+\frac{\partial}{\partial z}\left(k \frac{\partial T}{\partial z}\right)+q_{n e t}
$$

where $H$ is the mixing enthalpy, $T$ is the temperature, $k$ is the heat conductive coefficient. $q_{n e t}$ is the heat input during laser-MIG hybrid welding process.

The heat balance equation is:

$$
q_{n e t}=q_{A}+q_{L}+q_{D}-q_{E}-q_{R}-q_{C}
$$

where $q_{A}$ is the heat input from the electric arc; $q_{L}$ is the heat input from the laser; $q_{D}$ is the heat input from droplet; $q_{E}$ is the evaporation heat loss from the molten metal; $q_{R}$ is the heat loss by radiation; $q_{C}$ is the heat loss by convective heat exchange with the environment. The unite of these parameters is $\mathrm{J} / \mathrm{m}^{2} / \mathrm{s}$.

Momentum equation 


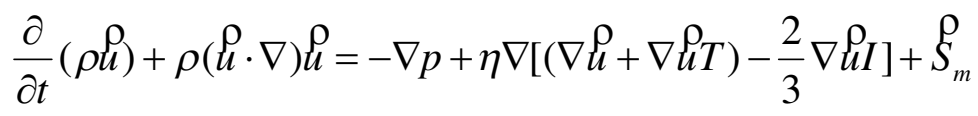

where $\mathbf{u}=(\mathrm{u}, \mathrm{v}, \mathrm{w}), \mathbf{S}_{\mathbf{m}}$ is the source term for momentum conservation.

\subsection{Driving forces}

The vapor recoil pressure $\left(P_{R}\right)$ of the gas is an important reason for the keyhole, which is mainly driven by the plasma generated at the bottom of the keyhole, and the liquid metal is expelled to both sides of the molten pool[23,24]. The equation of recoil pressure is expressed as follows:

$$
P_{R}=P_{0} \cdot \exp \left(L_{v} \frac{T-T_{b}}{R T T_{b}}\right)
$$

where $P_{0}$ is the ambient air pressure, $L_{v}$ is latent heat of vaporization, $T_{b}$ is the boiling temperature, and $R$ is the gas constant value.

The surface tension is one of the driving forces to increase the width of the molten pool. The temperature coefficient of the surface tension with the aluminum alloy liquid metal is negative[25]. The molten metal on the surface of the molten pool flows around the keyhole, resulting in a wide and shallow cross-section of the molten pool.

Marangoni force has great effect on the width of molten pool. Especially, when the temperature gradient coefficient of surface tension is negative, the surface tension of the liquid metal increases as the temperature decreases. Thus, the surface tension is low at the center of the molten pool, and it is high near the molten pool edge, which contributes to an outward flow and consequently a wider and shallower molten pool. The equation of Marangoni force is expressed as follows:

$$
\tau=\frac{d \gamma}{d T} \frac{d T}{d y}
$$

where $\tau$ is the Marangoni stress, $d \gamma / d T$ is temperature coefficient of surface tension and $d T / d y$ is the temperature gradient.

The buoyancy force is caused by the density variations. The variation of density in the molten pool induces the fluid rising in the hotter and less-dense region. At the same time, the fluid sinks in the cooler and denser region. The force is expressed by the following equation:

$$
F_{b}=\rho g \beta\left(T-T_{r e f}\right)
$$

where $\rho$ is the density of liquid metal, $\beta$ is the thermal expansion coefficient, and $T_{r e f}$ is 
arbitrarily selected reference temperature.

Electromagnetic force is named as Lorentz force, which effects on the heat and mass transfer in the molten pool and contributes to smaller width and deeper penetration. The equation is expressed as:

$$
F_{m}=\stackrel{\mu}{J} \times \ddot{B}
$$

where $J$ is the current density and $B$ is the Magnetic field.

Arc pressure contributes to smaller width and larger penetration. On the contrary, the plasma flow force leads to larger width and shallower penetration. The scheme diagram of driving force in the molten pool is as shown in Fig. 3.
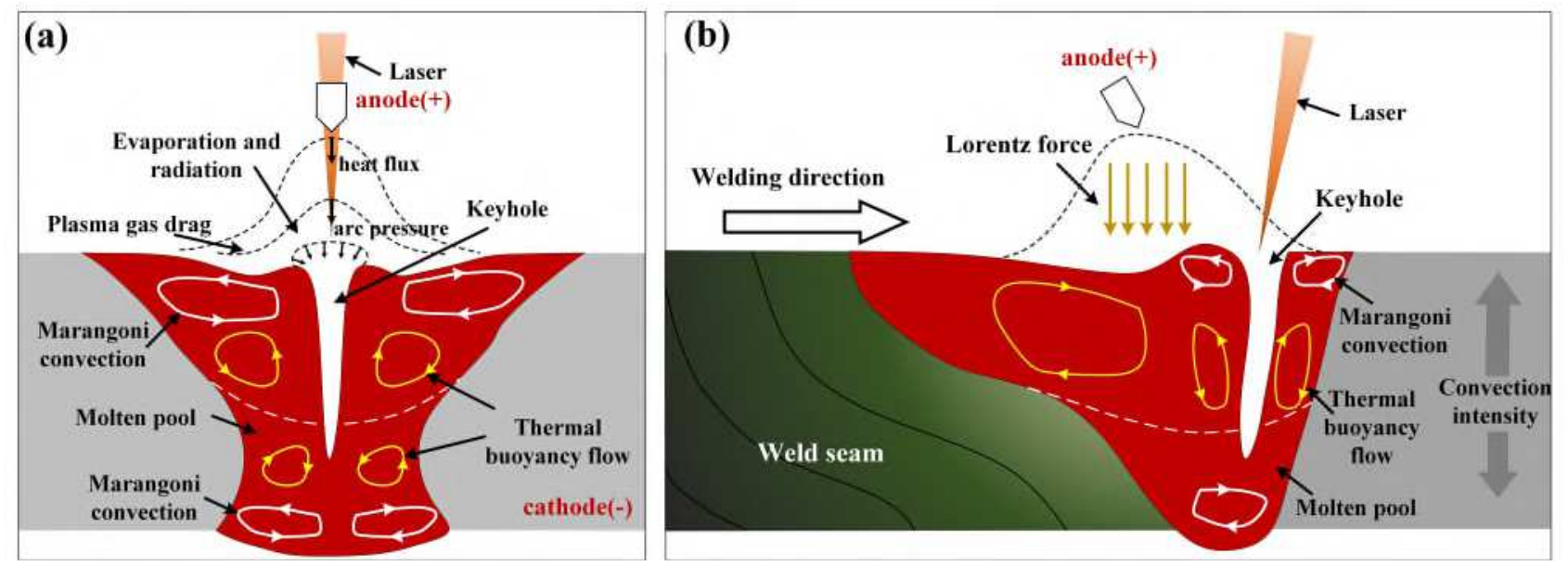

Fig. 3 The schematic diagram of driving force during laser-MIG hybrid welding. (a) In cross section. (b) In longitudinal section

\subsection{Condition of computational domain}

Considering the gas-liquid two-phase flow under the laser-MIG hybrid welding process, two domains of air and aluminum alloy are established, where the thickness of the air is $3 \mathrm{~mm}$ and the thickness of the aluminum alloy is $6 \mathrm{~mm}$, as shown in Fig. 4. The top of the air domain is selected as the inflow, the velocity is set to $0.1 \mathrm{~m} / \mathrm{s}$, and other three sides of the air domain as the outflow are set as an atmosphere. The remaining faces are set as walls. The geometric model is meshed to 487,978 tetrahedron elements. The thermo-physical properties of 6061 aluminum alloy and plasma are as shown in Table 3and Table 4, respectively. 

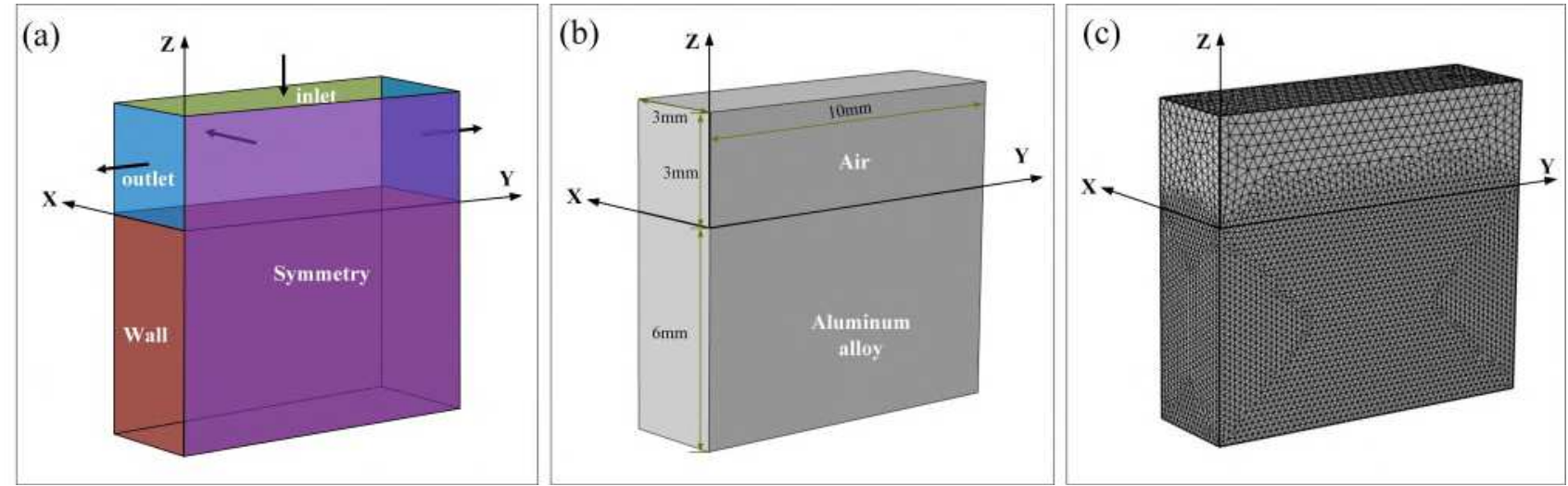

Fig. 4 The schematic diagram of computational domain

Table 3 Thermo-physical properties of 6061 aluminum alloy for simulation

\begin{tabular}{cccc}
\hline Physical property & Symbol & Value & $\mathrm{Unit}$ \\
\hline Mass density & $\rho$ & 2630 & $\mathrm{Kg} / \mathrm{m}^{3}$ \\
Thermal expansion coefficient & $\beta$ & $3.02 \times 10^{-5}$ & $1 / \mathrm{K}$ \\
Heat conductivity & $\lambda$ & 204 & $\mathrm{~W} /(\mathrm{m} \cdot \mathrm{K})$ \\
Solid temperature & $\mathrm{T}_{\mathrm{m}}$ & 858.13 & $\mathrm{~K}$ \\
Liquid temperature & $\mathrm{T}_{\mathrm{l}}$ & 923.15 & $\mathrm{~K}$ \\
Evaporation temperature & $\mathrm{T}_{\mathrm{b}}$ & 2790 & $\mathrm{~K}$ \\
Latent heat of fusion & $\mathrm{L}_{\mathrm{m}}$ & $5.03 \times 10^{5}$ & $\mathrm{~J} / \mathrm{kg}$ \\
Latent heat of evaporation & $\mathrm{L}_{\mathrm{v}}$ & $1.07 \times 10^{7}$ & $\mathrm{~J} / \mathrm{kg}$ \\
Special heat capacity of solid & $\mathrm{C}_{\mathrm{p}}$ & 900 & $\mathrm{~J} /(\mathrm{kg} \cdot \mathrm{K})$ \\
Special heat capacity of liquid & $\mathrm{C}_{\mathrm{l}}$ & 1200 & $\mathrm{~J} /(\mathrm{kg} \cdot \mathrm{K})$ \\
Surface tension & $\sigma$ & 0.99 & $\mathrm{~N} / \mathrm{m}$ \\
Surface emissivity & $\mathrm{A}_{\sigma}$ & $-1.3 \times 10^{-4}$ & $\mathrm{~N} /(\mathrm{m} \cdot \mathrm{K})$ \\
Ambient temperature & $\eta$ & 0.0045 & $\mathrm{~Pa} \cdot \mathrm{s}$ \\
Magnetic permeability & $\mathrm{T}_{\mathrm{ref}}$ & 300 & $\mathrm{H} / \mathrm{K}$ \\
\hline Demperature coefficient of surface tension & $\mathrm{N}_{2}$ & $1.26 \times 10^{-6}$ & $\mathrm{~K}$ \\
\hline
\end{tabular}

Table 4 Thermo-physical properties of plasma for simulation

\begin{tabular}{cccc}
\hline Physical property & Symbol & Value & Unit \\
\hline Mass density & $\rho_{\mathrm{g}}$ & 0.06 & $\mathrm{Kg} \cdot \mathrm{m}^{-3}$ \\
Special heat capacity & $\mathrm{C}_{\mathrm{g}}$ & 49 & $\mathrm{~J} \cdot \mathrm{Kg}^{-1}$
\end{tabular}




\begin{tabular}{cccc} 
Convection heat transfer coefficient & $\mathrm{h}_{\mathrm{g}}$ & 3.74 & $\mathrm{~W} \cdot \mathrm{m}^{-2} \cdot \mathrm{K}^{-1}$ \\
Dynamic viscosity & $\eta_{\mathrm{g}}$ & 0.0045 & $\mathrm{~Pa} \cdot \mathrm{s}$ \\
\hline
\end{tabular}

\subsection{Heat source}

Considering the laser and MIG heat sources during the welding process, a hybrid heat source model of a Gaussian rotating body heat source combination and a double ellipsoid heat source are selected for simulation, as shown in Fig. 5. The heating range of the MIG heat source is wider and the width of the weld seam is larger, thus the double ellipsoid heat source model is better reflect the shape of the molten pool[26]. The laser heat source acts on the surface of the workpiece and produces a keyhole effect, which plays a leading role in the welding process[27].

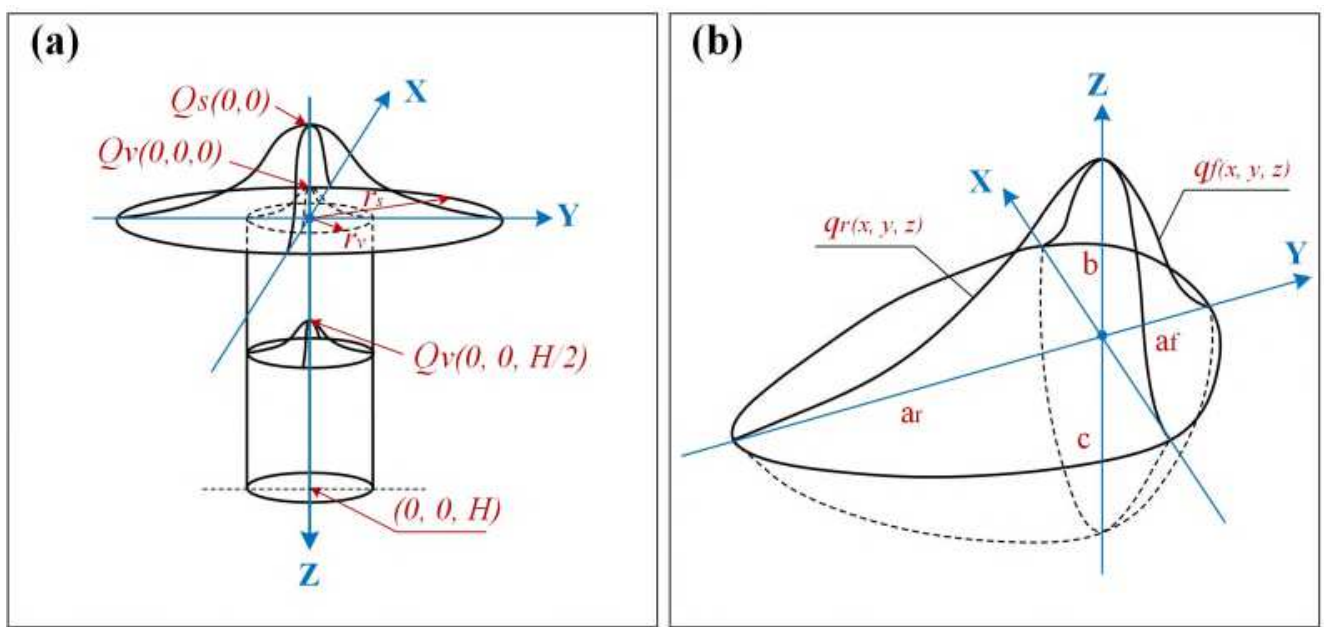

Fig. 5 The model of laser-MIG hybrid heat source. (a) Gaussian rotating body combined heat source model. (b)

Double ellipsoid heat source model

Since the weld under the laser heat source is nail-shaped in cross section, in order to better fit the simulation effect, the Gaussian rotating body heat source is selects as the laser heat source model, which mainly includes Gaussian surface heat source and Gaussian body heat source. The distribution functions of heat flux density are described as:

$$
\begin{array}{r}
q_{s}(x, y)=\frac{b Q_{s}}{\pi r_{s}^{2}} \exp \left(\frac{-b\left(x^{2}+y^{2}\right)}{r_{s}^{2}}\right) \\
q_{v}(x, y, z)=\frac{9 Q_{v}}{\pi R_{0} H(t)\left(1-e^{-3}\right)} \exp \left(\frac{-9\left(x^{2}+y^{2}\right)}{\lg \frac{H(t)}{z}}\right)
\end{array}
$$

where $q_{s}$ and $q_{v}$ are the heat flux distributions of Gaussian surface heat source and bulk heat 
source respectively, $b$ is the heat flux concentration coefficient, $Q_{s}$ and $Q_{v}$ represent the power of surface heat source and bulk heat source, $r_{s}$ and $R_{0}$ are the effective radius of action of the surface heat source and bulk heat source, respectively, $H(t)$ is the effective depth of action at which the body heat source changes over time. And the relationship between $Q_{s}$ and $Q_{v}$ is expressed as:

$$
Q_{V}+Q_{S}=\eta_{L} P
$$

where $P$ is the laser power and $\eta_{L}$ is the thermal efficiency of the laser.

The double ellipsoid heat source model is described as:

$$
\begin{gathered}
q_{f}(x, y, z)=\frac{6 \sqrt{3}\left(f_{f} \eta_{A} I U\right)}{a_{f} b c \pi \sqrt{\pi}} \exp \left(-\frac{3 x^{2}}{a_{f}{ }^{2}}-\frac{3\left(y-v_{0} t\right)^{2}}{b^{2}}-\frac{3 z^{2}}{c^{2}}\right), y \geq 0 \\
q_{r}(x, y, z)=\frac{6 \sqrt{3}\left(f_{r} \eta_{A} I U\right)}{a_{r} b c \pi \sqrt{\pi}} \exp \left(-\frac{3 x^{2}}{a_{r}{ }^{2}}-\frac{3\left(y-v_{0} t\right)^{2}}{b^{2}}-\frac{3 z^{2}}{c^{2}}\right), y<0 \\
f_{f}+f_{r}=2
\end{gathered}
$$

In the formula, $q_{r}$ and $q_{f}$ are the heat source densities of the two ellipsoids in the double ellipsoid heat source model, $f_{f}$ and $f_{r}$ are the heat distribution coefficients of the two ellipsoids, and $I$, $U$ and $v_{0}$ are the arc current, voltage and Welding speed, $a_{f}, a_{r}, b$ and $c$ are heat source model parameters. $\eta A$ is the arc thermal efficiency.

\subsection{Experiment validation}

According to the heat-fluid coupling solution process of the laser-MIG hybrid welding process, the numerical simulation calculation is carried out. After the relevant parameters of the model are corrected, the results of heat source verification result for 1\# is shown in Fig. 6. Comparing the weld cross section with the weld simulation results, it is seen that the macroscopic appearances of $1 \#$ is basically symmetrical, so the modified hybrid heat source model can be used for the subsequent simulation calculation of the temperature field and flow field of laser-MIG hybrid welding. 


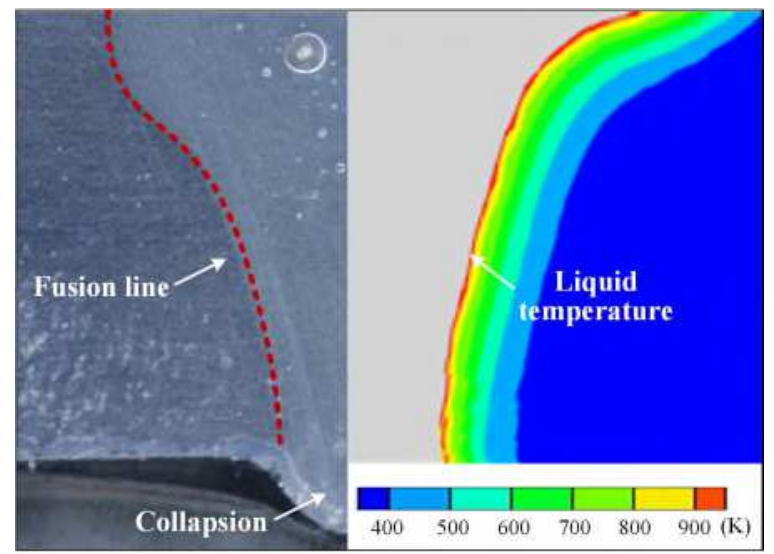

Fig. 6 Comparison of the experimental result and simulation result

\subsection{Droplet transition model}

The mass, energy and momentum brought by the droplet transition behavior, which impacts on the temperature field and flow field of the molten pool as well as the dynamic evolution behavior of the keyhole. In this model, the boundary at the entrance in the geometric model is set as the position where the droplet grows up gradually. As the droplet grows up completely, it falls into the molten pool by free fall. Based on the results observed by the high-speed camera, the droplet transition period of $0.06 \mathrm{~s}$ was set, and the wire moving speed was set the same as the laser heat source moving speed. The geometric model and boundary conditions of the droplet transition are shown in Fig. 7.

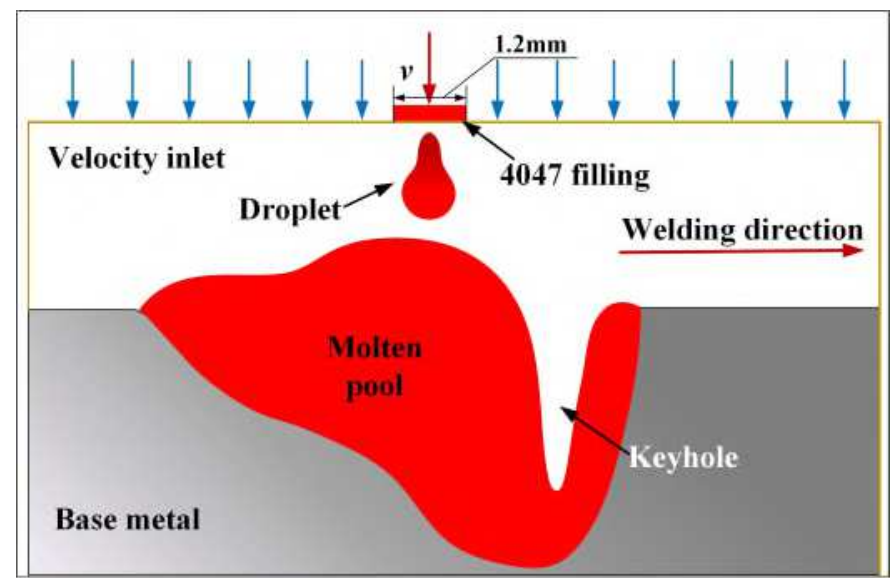

Fig. 7 Geometric and boundary conditions of droplet transition model

\section{Results and analysis}

\subsection{High-speed camera results}

In order to facilitate the analysis of the droplet transfer behavior in the laser-MIG hybrid welding process, the high-speed camera results of $1 \#$ parameter $(\mathrm{P}=4.0 \mathrm{~kW}, \mathrm{I}=90 \mathrm{~A}, \mathrm{v}=1.2 \mathrm{~m} / \mathrm{min})$ 
were taken, and a picture was extracted every $2 \mathrm{~ms}$ for observation and analysis, as shown in Fig. 8. At $t_{0}$, the droplet just started to contact the molten pool. Due to inertia, the center of gravity of the molten droplet shifted along the side opposite to the welding direction. The contact surface gradually increased under the action of the surface tension of the molten pool and gravity, the molten droplet was completely separated from the end of the welding wire at $t_{0}+6 \mathrm{~ms}$. With the falling of the molten droplet, the molten droplet gradually merged into the molten pool. At $\mathrm{t}_{0}+10 \mathrm{~ms}$, the molten droplet completely merged into the molten pool. The front of the molten pool formed a keyhole under the action of the laser. Owing to the impact and momentum brought by the droplet drop, a depression appeared in the front of the liquid molten pool at $\mathrm{t}_{0}+12 \mathrm{~ms}$, and the diameter of the keyhole was also significantly reduced.

It is seen that the droplet transition behavior has a very important influence on the stability of the molten pool and the volatility of the keyhole. The external momentum and energy brought by the droplet transition promote the flow of the molten pool, which is beneficial for the beneficial elements of the molten droplet. The elements are fully mixed with the molten pool and promote the escape of bubbles. In the process of droplet transition, the back wall of the keyhole is squeezed due to the impact force of the droplet on the molten pool. The opening of the keyhole is suppressed, meanwhile, the laser energy is affected to the bottom of the keyhole, which results a smaller keyhole. 

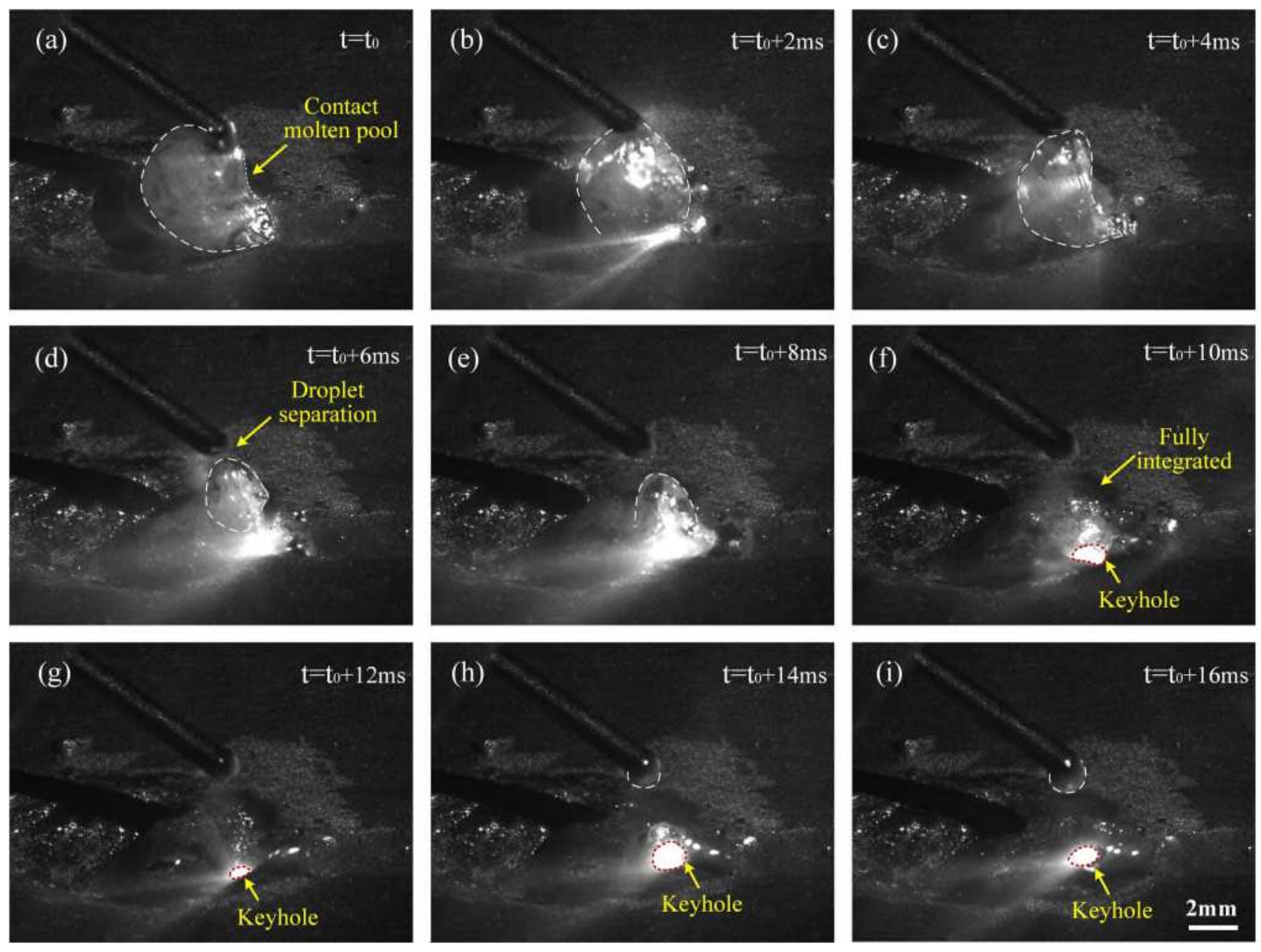

Fig. 8 High-speed camera photos in the droplet transition process

\subsection{Effect of droplet transition on thermal-fluid coupling field distribution}

In order to study the effect of droplet transition on the temperature field and flow field of the molten pool, under 2\# parameter $(\mathrm{P}=4.0 \mathrm{~kW}, \mathrm{I}=100 \mathrm{~A}, \mathrm{v}=1.2 \mathrm{~m} / \mathrm{min})$, the droplet between $64.8 \mathrm{~ms}$ and $68.8 \mathrm{~ms}$ is selected. The results of the transition simulation were analyzed, and the method of adding and not adding the droplet transition was used to analyze the influence of the droplet transition behavior on the morphology of the keyhole. The simulation results of the temperature field are shown in Fig. 9 and Fig. 10, respectively.

The droplet transition process causes pressure on the back wall of the keyhole, so the angle of the keyhole back wall changes, as shown in Fig. 9. At $\mathrm{t}=64.8 \mathrm{~ms}$, the molten droplet was contracted and hung above the molten pool under the action of electromagnetic force, the back of the molten pool was slightly dented by the action of the arc force. At $\mathrm{t}=65.6 \mathrm{~ms}$, the droplet contacted the back of the molten pool, the momentum by the falling droplet caused an impact on the molten pool, and 
the back wall of the keyhole was squeezed by the liquid at the back of the molten pool. At $\mathrm{t}=67.2 \mathrm{~ms}$, the back wall of the keyhole bulged due to the squeezing effect of the droplets falling into the molten pool, and the direction pointed to the inside of the keyhole. At $\mathrm{t}=68.0 \mathrm{~ms}$, owing to the squeezing of the molten metal in the rear molten pool and the filling of the molten pool, the angle of the lower part of the back wall of the keyhole was changed, which facilitated the laser irradiation into the keyhole and brought a greater depth of the keyhole. At $\mathrm{t}=68.8 \mathrm{~ms}$, the back wall of the keyhole continued to be affected by the fusion of the molten droplets to the back of the molten pool, and the lower part of the back wall of the keyhole had a boss pointing to the inside, which was not conducive to the laser irradiation to the bottom of the keyhole, thus affected the absorption of laser energy in molten pool.

Compare with added droplet transfer, the surface morphology of the back of the molten pool without droplet is smoother, as shown in Fig. 10. It is found that the depth of the keyhole is the same, but the back wall of the keyhole changes greatly. The increase in the volume of the rear part causes a slight depression in the lower part of the rear wall of the keyhole.

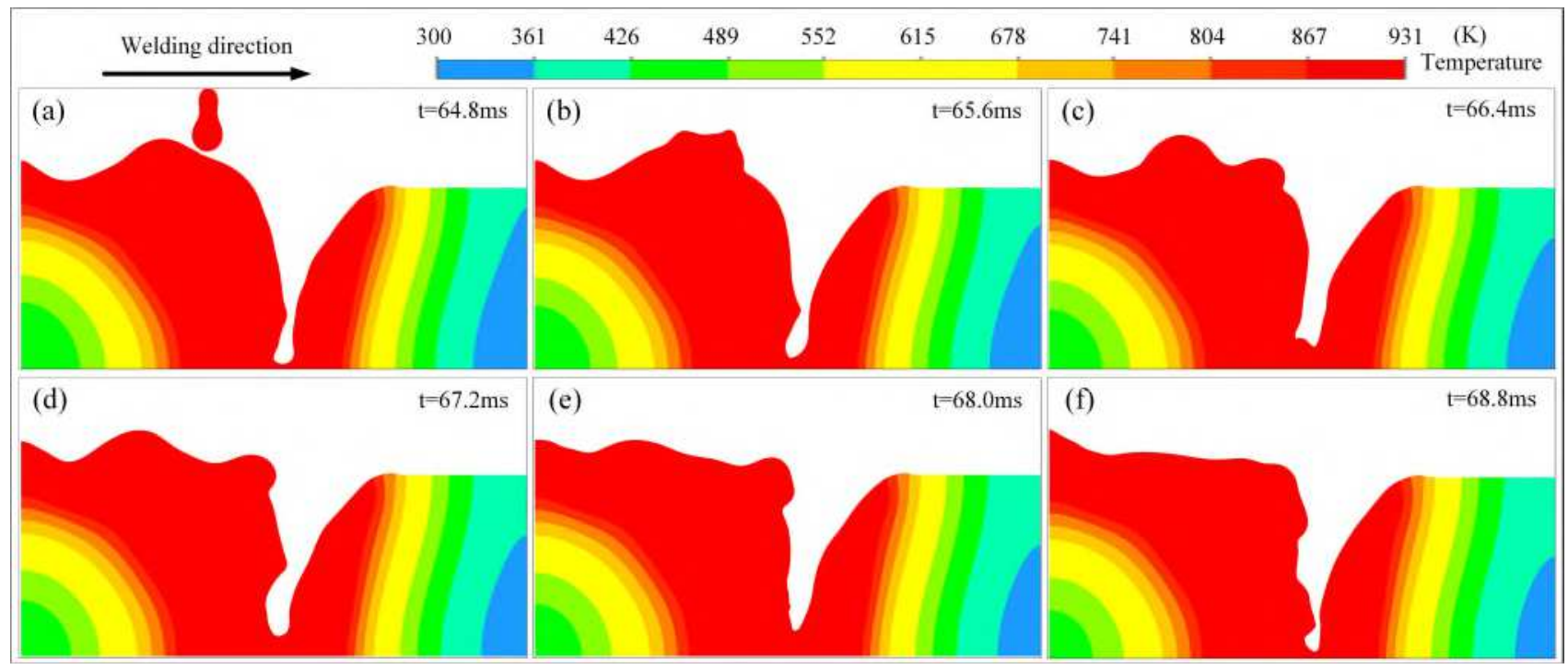

Fig. 9 Distribution of temperature field in molten pool during droplet transition. (a) $t=64.8 \mathrm{~ms}$. (b) $t=65.5 \mathrm{~ms}$. (c) $t=66.4$ ms. (d) $t=67.2$ ms. (e) $t=68.0$ ms. (f) $t=68.8 \mathrm{~ms}$ 


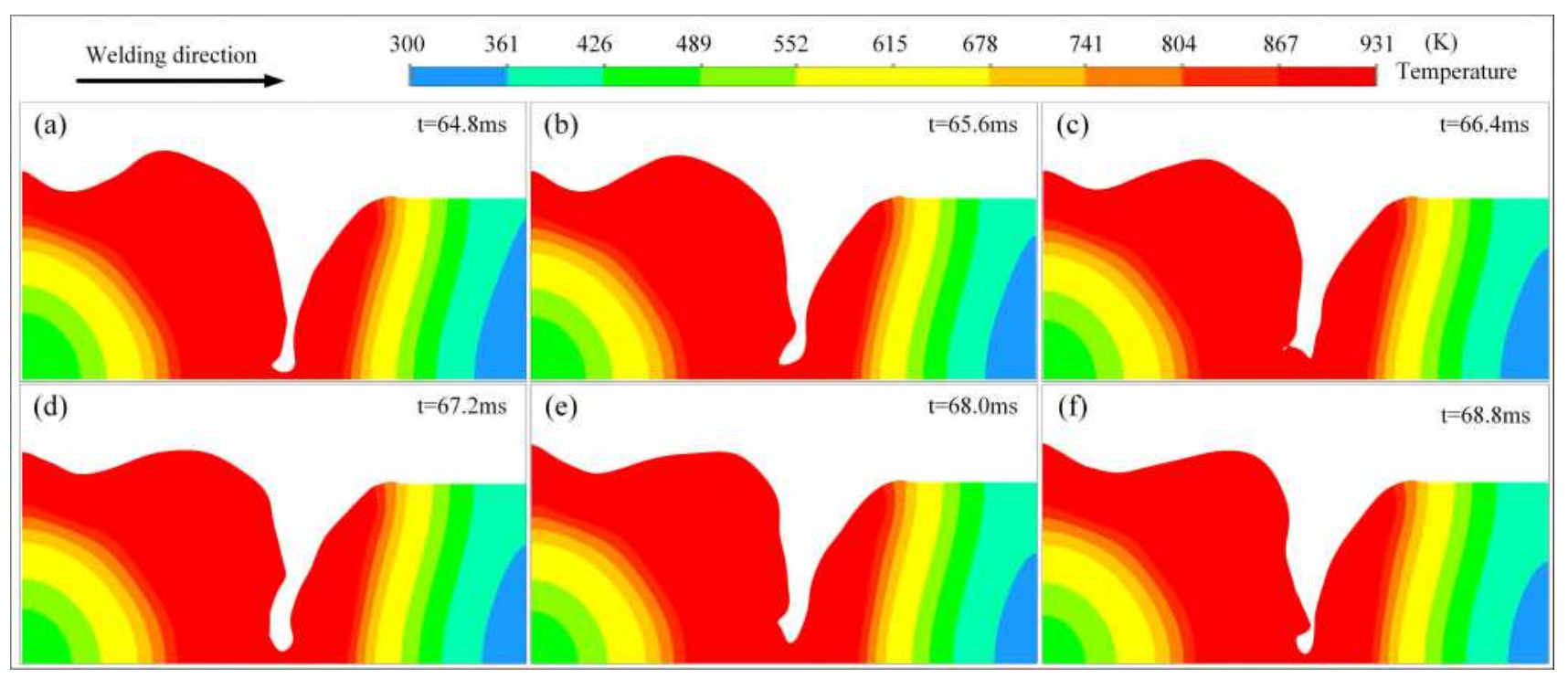

Fig. 10 Distribution of temperature field in molten pool without droplet transition. (a) $t=64.8 \mathrm{~ms}$. (b) $\mathrm{t}=65.5 \mathrm{~ms}$.

$$
\text { (c) } \mathrm{t}=66.4 \text { ms. (d) } \mathrm{t}=67.2 \mathrm{~ms} \text {. (e) } \mathrm{t}=68.0 \mathrm{~ms} \text {. (f) } \mathrm{t}=68.8 \mathrm{~ms}
$$

Because the molten droplet brings external momentum to the molten pool, the flow field distribution of the molten pool also changes. The simulation results of the flow field are shown in Fig. 11 and Fig. 12, respectively.

It is seen from Fig. 11 that the flow velocity inside the molten pool is very small, the flow velocity on the surface of the molten pool and the wall of the keyhole is relatively large. The flow velocity on the surface of the molten pool varies from $0.7 \mathrm{~m} / \mathrm{s}$ to $1.1 \mathrm{~m} / \mathrm{s}$, the flow velocity at the bottom of the keyhole varies greatly, about $0.4 \mathrm{~m} / \mathrm{s}$ to $1.4 \mathrm{~m} / \mathrm{s}$. At t=64.8 ms, the molten droplet had not yet fallen into the molten pool. At this time, the fluid in the front of the molten pool flowed upward along the front wall of the keyhole, and the fluid at the rear of the molten pool flowed down in a clockwise direction in front of the molten pool. It flowed upward along the back wall of the keyhole and extends backward under the action of Marangoni circulation on the surface of the molten pool. A clockwise vortex appeared in the fluid below the back of the molten pool. At $\mathrm{t}=65.6$ $\mathrm{ms}$, the transition of the droplet brought an impact to the back of the molten pool, so the flow direction of the fluid in the bottom area of the keyhole was from the back to the front of molten pool. Meanwhile, the droplet completely fell into the molten pool, the impact force brought by the droplet was relatively large, and the maximum velocity reached $2.05 \mathrm{~m} / \mathrm{s}$.

For the flow field distribution of the molten pool without added droplet transition, the flow field distribution is basically the same as that of the added droplet transition, but its peak velocity at 
different times is less than the added droplet transition, mainly because the droplet transition brings about External momentum[28]. The change of the shape of the keyhole by the droplet transition is mainly achieved by promoting the downward flow of the fluid at the back of the molten pool to fill the depression below the back wall of the keyhole, thus squeezing the back wall of the keyhole. When convex shape is appeared on the back wall of the keyhole, which affects the heating effect of the laser on the molten pool, the back wall of the keyhole will adjust the angle under the thermal capillary force to maintain the absorption of the laser by the molten pool.
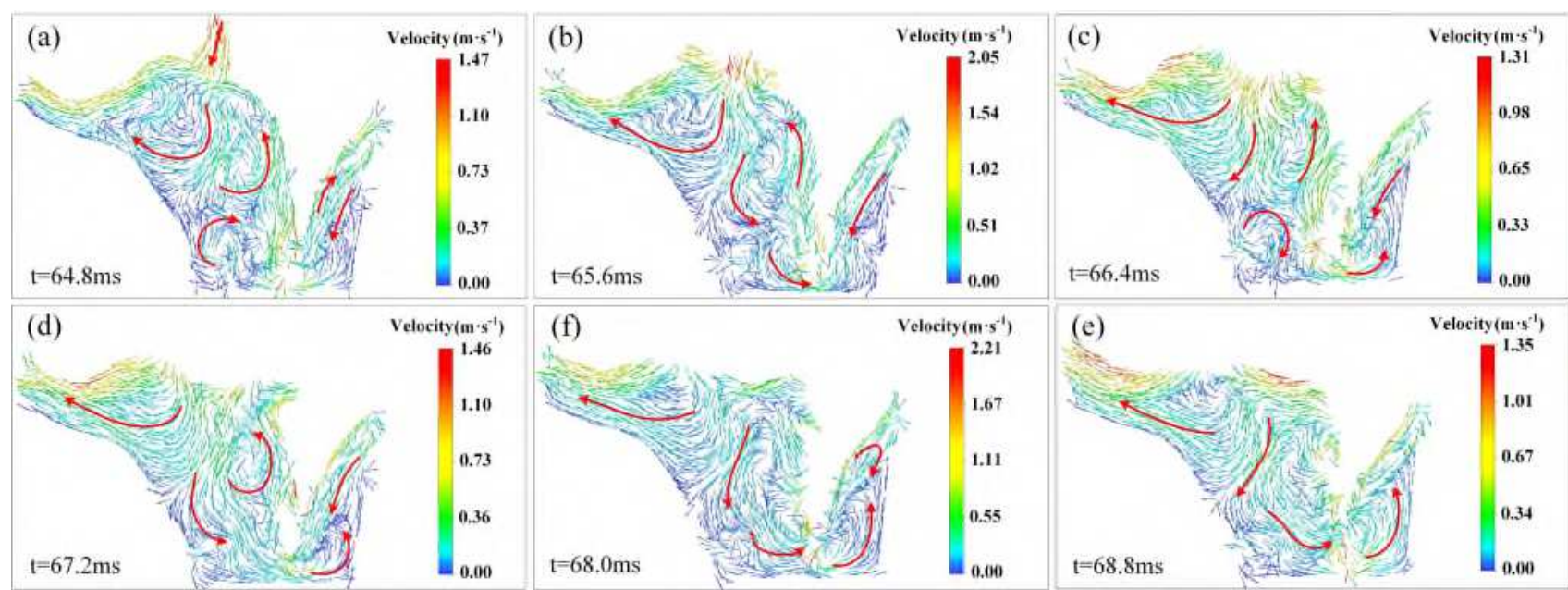

Fig. 11 Distribution of flow field in molten pool during droplet transition. (a) $t=64.8 \mathrm{~ms}$. (b) $\mathrm{t}=65.5 \mathrm{~ms}$. (c) $t=66.4$ ms. (d) $t=67.2$ ms. (e) $t=68.0$ ms. (f) $t=68.8 \mathrm{~ms}$

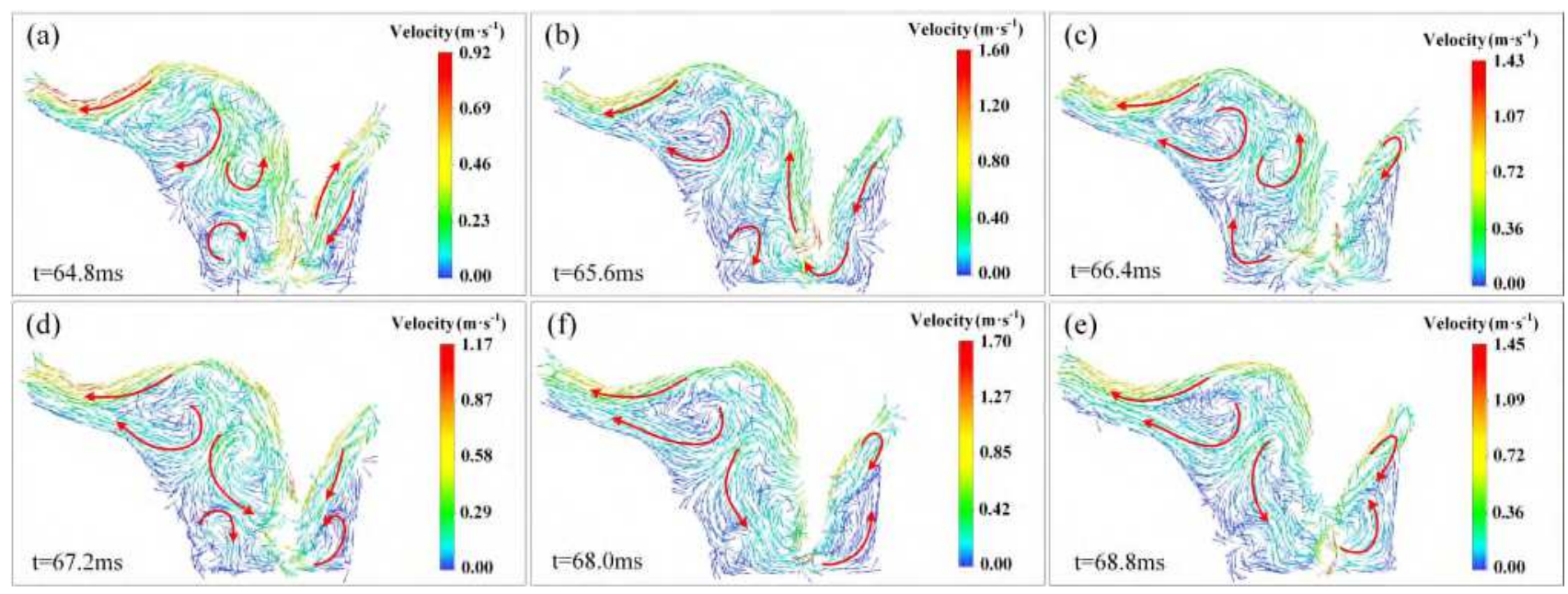

Fig. 12 Distribution of flow field in molten pool without droplet transition. (a) $t=64.8 \mathrm{~ms}$. (b) $\mathrm{t}=65.5 \mathrm{~ms}$.

(c) $t=66.4$ ms. (d) $t=67.2$ ms. (e) $t=68.0$ ms. (f) $t=68.8 \mathrm{~ms}$

\subsection{Effect of droplet on keyhole induced pore}

Fig. 13 shows that the trajectory of the bubble formed during the droplet transition under $2 \#$ parameter $(\mathrm{P}=4.0 \mathrm{~kW}, \mathrm{I}=100 \mathrm{~A}, \mathrm{v}=1.2 \mathrm{~m} / \mathrm{min})$. It is seen that the droplet transition has an important 
influence on the stability of the keyhole, and it is easy to bring external bubbles when falling into the molten pool. Before the molten droplet fell into the molten pool, the maximum speed of the free fall reached $2.262 \mathrm{~m} / \mathrm{s}$, which brought external momentum and energy to the molten pool. At $\mathrm{t}=66.4 \mathrm{~ms}$, the molten droplet fell into the molten pool forming a gap, and the movement direction of the surrounding fluid was a counterclockwise vortex, the vortex velocity was $0.39 \sim 0.78 \mathrm{~m} / \mathrm{s}$; at $\mathrm{t}=67.2$ ms, an irregular bubble formed on the surface at the back of the molten pool. The flow direction of the fluid was still a counterclockwise vortex, and the moving speed is reduced; at $t=67.6 \mathrm{~ms}$, the liquid bridge above the bubble was broken, and the bubble escaped from the molten pool. The flow direction of the fluid around the bubble was clockwise circulation, and the maximum surface velocity caused by the liquid bridge fracture reached $2.805 \mathrm{~m} / \mathrm{s}$. It can be seen that the droplet transition brings smaller bubbles to the surface of the molten pool, and escapes the surface of the molten pool under the action of the surrounding circulation. The circulation velocity is slightly higher than that of the surrounding fluid, and the bubbles are easy to escape.
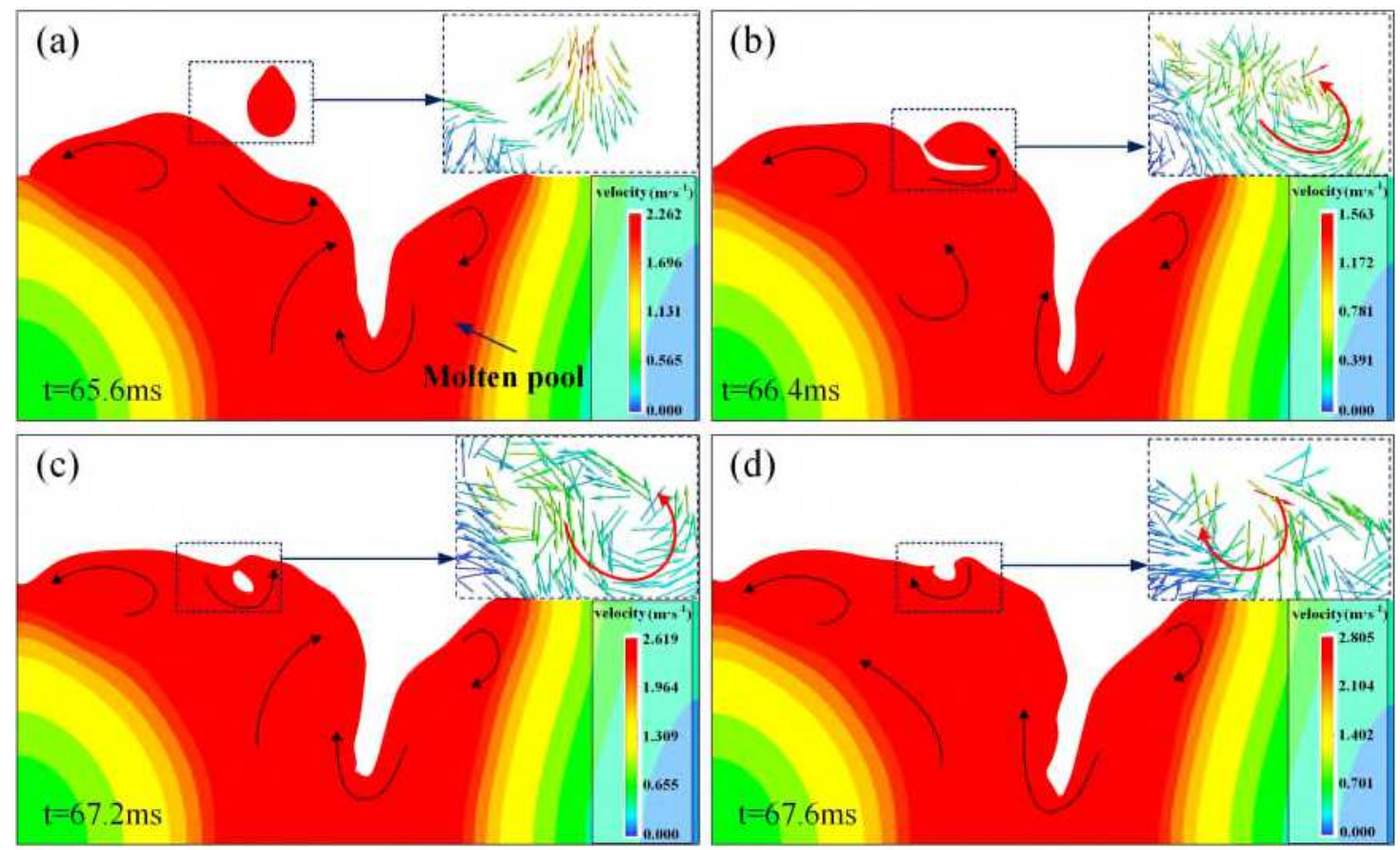

Fig. 13 The trajectory of the bubble formed during the droplet transition. (a) $t=65.6 \mathrm{~ms}$. (b) $t=66.4 \mathrm{~ms}$.

$$
\text { (c) } t=67.2 \text { ms. (d) } t=67.6 \mathrm{~ms}
$$

The forces acting on the bubbles in the molten pool are buoyancy force $F_{b}$, gravity $G$, the fluid driving force $F_{d}$ and viscous force $F_{V}$, as shown in Fig. 14(a). Because most of the bubbles are very small with the diameter of tens of microns, $F_{d}$ is the main force. The bubbles move in the molten 
pool under the driving force of the molten pool fluid[29]. Fig. 14(b) shows that the smaller bubbles move downward along the solidification interface under the action of fluid flow in the middle of the molten pool, and upward along the back wall of keyhole under the action of Marangoni circulation at the back of the molten pool. Finally, the smaller bubbles escape from the molten pool. For the large bubbles formed in the molten pool, which are mainly affected by $F_{b}$ and easy to escape from the molten pool.
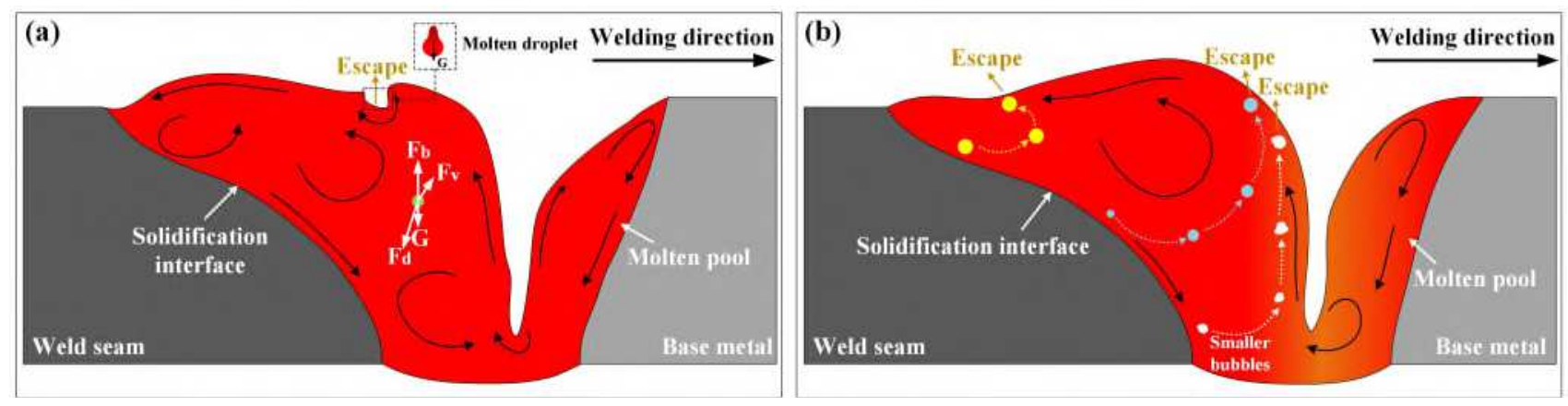

Fig. 14 (a) The schematic diagram of the bubble trajectory during the droplet transition. (b) The schematic diagram of the bubbles trajectory in different positions of the molten pool

\section{Conclusions}

In this investigation, high-speed camera was used to observe the droplet transition of laser-MIG hybrid welding for aluminum alloy. The thermal-fluid coupling model was established to study the effect of droplet transfer on the shape of the molten pool. Based on the experimental and simulation results, the conclusions are drawn as follows:

(1) The process of the droplet falling into the molten pool hinders the formation of the keyhole, and the keyhole opening is the smallest when the droplets are in contact with the molten pool.

(2) The mass, energy and momentum brought by the droplet transition behavior, which changes the temperature field and flow field of the molten pool. In addition, the maximum velocity of molten pool is bigger than that without droplet.

(3) The changes of the keyhole morphology are mainly behaved as the squeezing of the back wall of the keyhole. Additionally, the appearance of a boss on the keyhole wall affects the absorption of laser energy by the molten pool, thus affects the depth of keyhole.

(4) The droplet transition is easy to bring bubbles, which are drawn into the molten pool by the counterclockwise vortex formed on the upper surface of the molten pool. After that, under the 
combined action of the thermal buoyancy and the clockwise vortex formed on the upper surface of the molten pool, the bubbles is easy to escape the molten pool.

\section{Declaration}

\section{Funding}

The authors did not receive support from any organization for the submitted work.

\section{Conflicts of interest/Competing interests}

The authors have no conflicts of interest to declare that are relevant to the content of this article.

\section{Availability of data and material}

All data generated or analyzed during this study are included in this published article.

\section{Code availability}

Not applicable.

\section{Ethics approval}

The content in this article does not violate the ethics.

\section{Consent to participate}

All authors in this article have informed consent to participate in this study.

\section{Consent for publication}

All authors and their institutions agree to publish this article in The International Journal of Advanced Manufacturing Technology.

\section{Authors' contributions}

Yue Li: investigation, experiment, data analysis, original manuscript writing, manuscript revising. Yanqiu Zhao: validation, supervision, funding acquisition. Xudong Zhou: investigation, experiment. Xiaohong Zhan: manuscript revising.

\section{References}

[1] Mediratta R, Ahluwalia K, Yeo SH (2016) State-of-the-art on vibratory finishing in the aviation industry: an industrial and academic perspective. Int J Adv Manuf Technol 85:415-429.

[2] Samal P, Vundavilli PR, Meher A, Mahapatra MM (2020) Recent progress in aluminum metal matrix composites: A review on processing, mechanical and wear properties. J Manuf Process 59:131-152. 
[3] Alahmari AM, Darwish S, Ahmed, N (2016) Laser beam micro-milling (LBMM) of selected aerospace alloys. Int J Adv Manuf Technol 86:2411-2431.

[4] Dursun T, Soutis C (2014) Recent developments in advanced aircraft aluminium alloys. Mater Des 56:862-871.

[5] Zhou SJ, Ling WL, Ma WP, Gao QY, Lu WH, Zhan XH (2019) The pores formation mechanism in the laser-MIG hybrid welded joint of mild steel. Mater Res Express 6:095803.

[6] Chen S, Zhao YQ, Tian SH, Gu YZ, Zhan XH (2020) Study on keyhole coupling and melt flow dynamic behaviors simulation of 2219 aluminum alloy T-joint during the dual laser beam bilateral synchronous welding. J Manuf Process 60:200-212.

[7] Xu GX, Zheng ZQ, Cao QN, Hu QX, Li L, Guo QH, Du BS (2019) Numerical and experimental investigation on weld formation during laser+MIG hybrid fillet welding of aluminum alloy in horizontal position. Int J Adv Manuf Technol 102:2683-2694.

[8] Examilioti TN, Kashaev N, Enz J, Klusemann B, Alexopoulos ND (2020) On the influence of laser beam welding parameters for autogenous AA2198 welded joints. Int J Adv Manuf Technol 110:2079-2092.

[9] Guan Q, Long J, Yu P, Jiang S, Huang W, Zhou J (2019) Effect of steel to aluminum laser welding parameters on mechanical properties of weld beads. Opt Laser Technol 111:387-394.

[10]Zhao YQ, Zhan XH, Gao QY, Chen S, Kang Y (2020) Research on the Microstructure Characteristic and Tensile Property of Laser-MIG Hybrid Welded Joint for 5A06 Aluminum Alloy. Met Mater Int 26(3):346-359.

[11]Hammad A, Churiaque C, Sanchez-Amaya JM, Abdel-Nasser Y (2021) Experimental and numerical investigation of hybrid laser arc welding process and the influence of welding sequence on the manufacture of stiffened flat panels. J Manuf Process 61:527-538.

[12]Zhang C, Gao M, Wang D, Yin J, Zeng X (2017) Relationship between pool characteristic and weld porosity in laser arc hybrid welding of AA6082 aluminum alloy. J Mater Process Technol 240:217-222.

[13]Zhan X, Zhao Y, Liu Z, Gao Q, Bu H (2018) Microstructure and porosity characteristics of 5A06 aluminum alloy joints using laser-MIG hybrid welding. J Manuf Process 35:437-445.

[14] Yan J, Gao M, Li G, Zhang C, Zeng X, Jiang M (2013) Microstructure and mechanical properties of laser-MIG hybrid welding of 1420 Al-Li alloy. Int J Adv Manuf Technol 
66:1467-1473.

[15]Liu F, Tan C,Wu L, Gong X, Chen B, Song X, Zhao H, Wang G (2020) Influence of waveforms on Laser-MIG hybrid welding characteristics of 5052 aluminum alloy assisted by magnetic field. Opt Laser Technol 132:106508.

[16]Faraji AH, Moradi M, Goodarzi M, Coluccid P, Maletta C (2017) An investigation on capability of hybrid Nd:YAG laser-TIG welding technology for AA2198 Al-Li alloy. Opt Lasers Eng $96: 1-6$

[17]Liu S, Zhang F, Dong S, Zhang H, Liu F (2018) Characteristics analysis of droplet transfer in laser-MAG hybrid welding process. Int J Heat Mass Transf 121:805-811.

[18]Zhang F, Liu S, Liu F, Zhang H (2019) Stability evaluation of laser-MAG hybrid welding process. Opt Laser Technol 116:284-292.

[19]Lu FG, Li XB, Li ZG, Tang XH, Cui CH (2015) Formation and influence mechanism of keyhole-induced porosity in deep-penetration laser welding based on 3D transient modeling. Int J Heat Mass Transf 90:1143-1152.

[20]Xu G, Li L, Wang H, Li P, Guo Q, Hu Q, Du B (2019) Simulation and experimental studies of keyhole induced porosity in laser-MIG hybrid fillet welding of aluminum alloy in the horizontal position. Opt Laser Technol 119:105667.

[21]Cunningham R, Zhao C, Parab N, Kantzos C, Pauza J, Fezzaa K, Sun T, Rollett AD (2019) Keyhole threshold and morphology in laser melting revealed by ultrahigh-speed x-ray imaging. Science 363:849-852.

[22] Ye XH, Chen X (2002) Three-dimensional modelling of heat transfer and fluid flow in laser full-penetration welding. J Phys D: Appl Phys 35:1049-1056.

[23] Semak V, Matsunawa A (1997) The role of recoil pressure in energy balance during laser materials processing. J Phys D: Appl. Phys 30:2541-2552.

[24]Pang S, Chen X, Shao X, Gong S, Xiao J (2016) Dynamics of vapor plume in transient keyhole during laser welding of stainless steel: Local evaporation, plume swing and gas entrapment into porosity. Opt Lasers Eng 82:28-40.

[25]Cho WI, Na SJ, Cho MH, Lee SJ (2010) Numerical study of alloying element distribution in CO2 laser-GMA hybrid welding. Comput Mater Sci 49:792-800.

[26]Wang Y, Tsai HL (2001) Impingement of filler droplets and weld pool dynamics during gas 
metal arc welding process. Int J Heat Mass Transf 44:2067-2080.

[27]Lankalapalli KN, Tu JF, Gartner M (1996) A model for estimating penetration depth of laser welding processes. Phys D: Appl Phys 29:1831-1841.

[28]Liu S, Zhang F, Dong S, Zhang H, Liu F (2018) Characteristics analysis of droplet transfer in laser-MAG hybrid welding process. Int J Heat Mass Transf 121:805-811.

[29] Zhang C, Gao M, Wang DZ, Yin J, Zeng XY (2017) Relationship between pool characteristic and weld porosity in laser arc hybrid welding of AA6082 aluminum alloy. J Mater Proc Technol 240:217-222. 


\section{Figures}

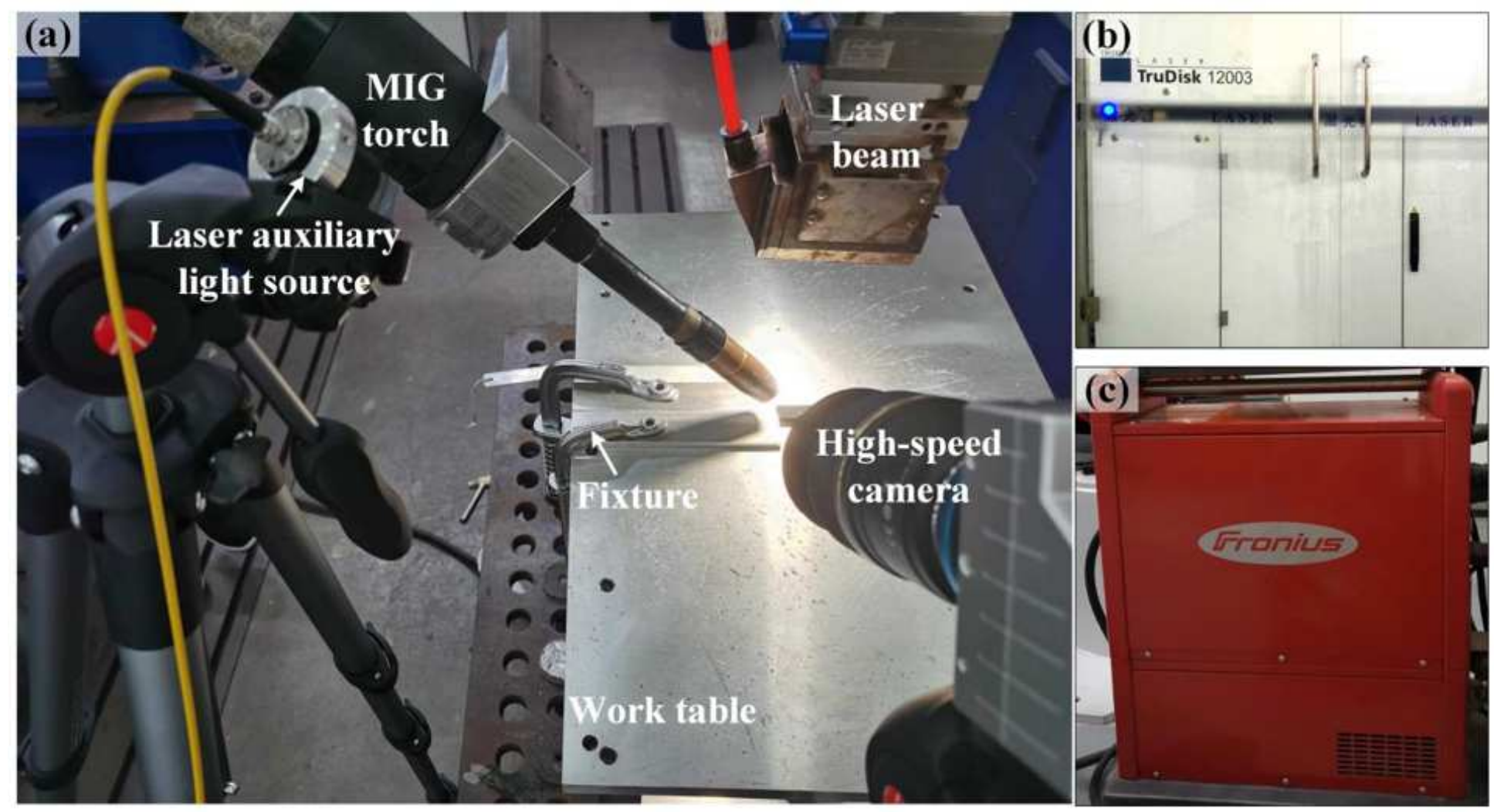

\section{Figure 1}

Experimental setup of 6061 aluminum alloy laser-MIG hybrid welding. (a) Equipment and work table. (b) Disc laser. (c) Fronius welder
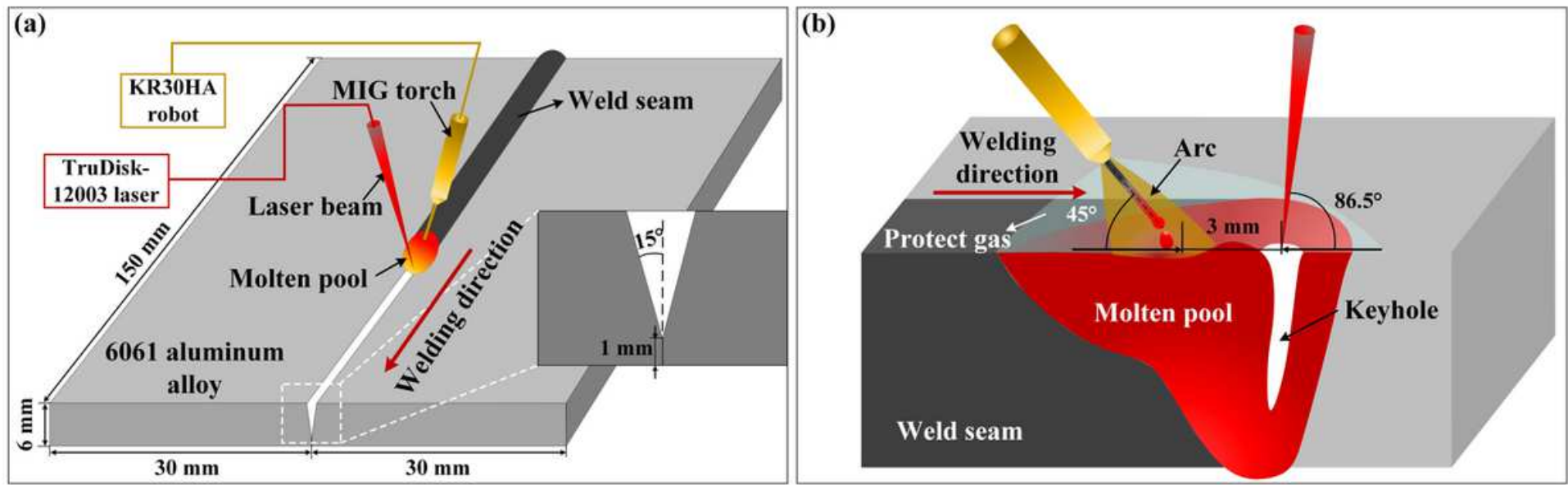

Figure 2

Schematic diagram of laser-MIG hybrid welding. (a) Welding. (b)Molten pool morphology 

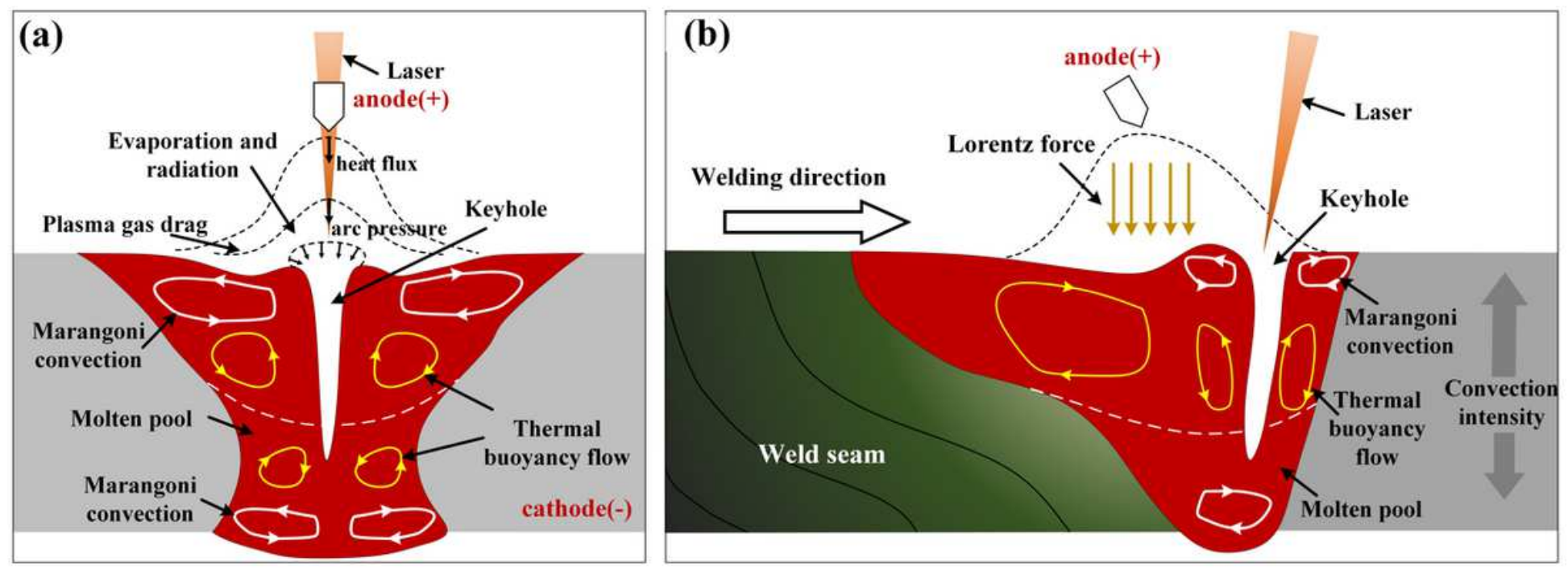

\section{Figure 3}

The schematic diagram of driving force during laser-MIG hybrid welding. (a) In cross section. (b) In longitudinal section
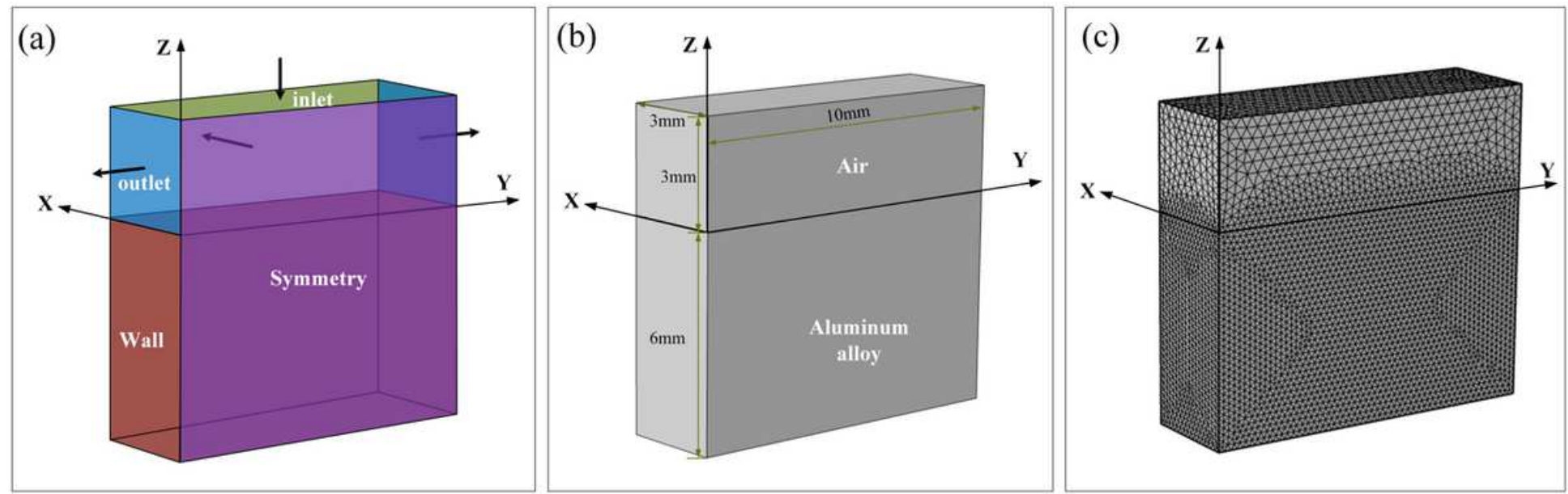

Figure 4

The schematic diagram of computational domain 

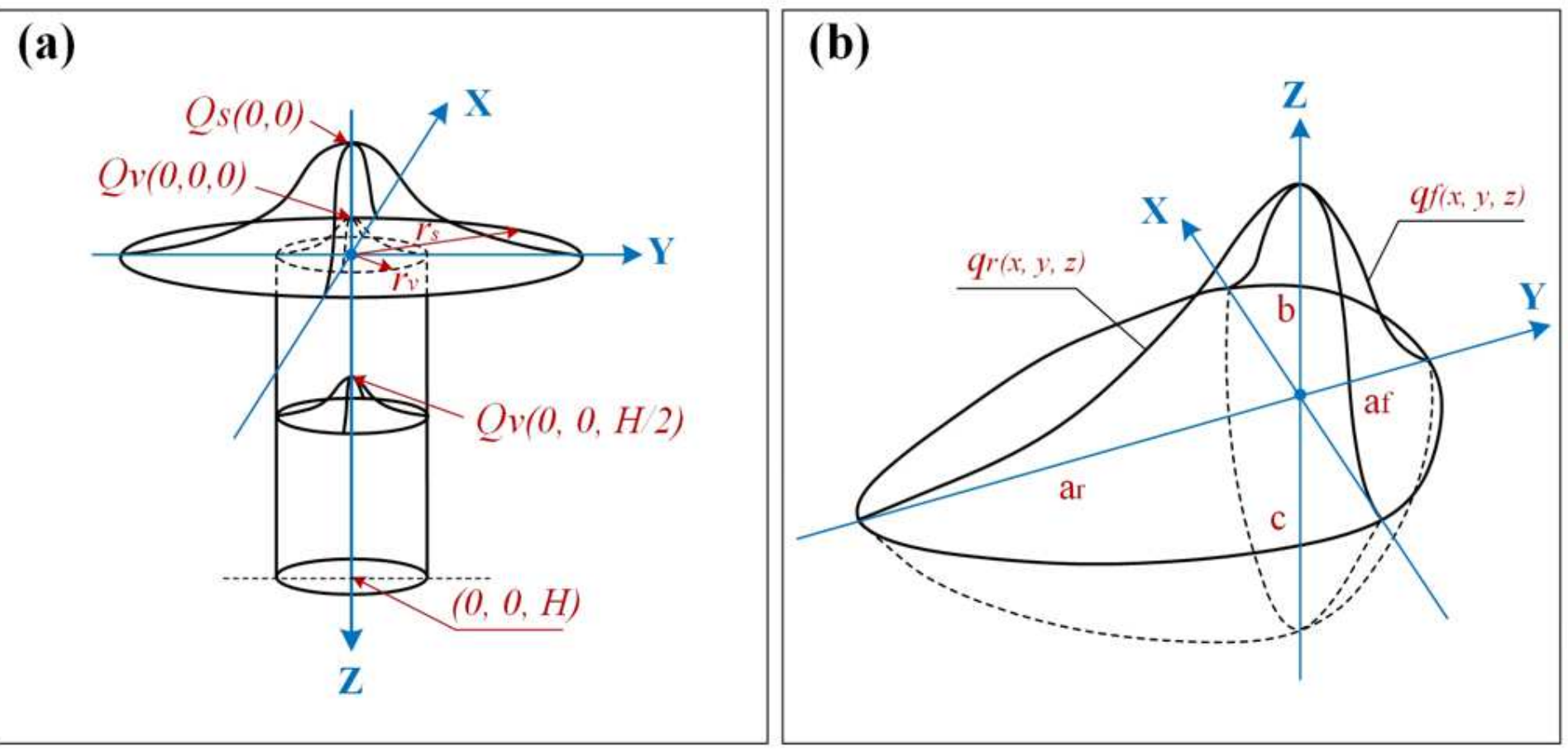

Figure 5

The model of laser-MIG hybrid heat source. (a) Gaussian rotating body combined heat source model. (b) Double ellipsoid heat source model 


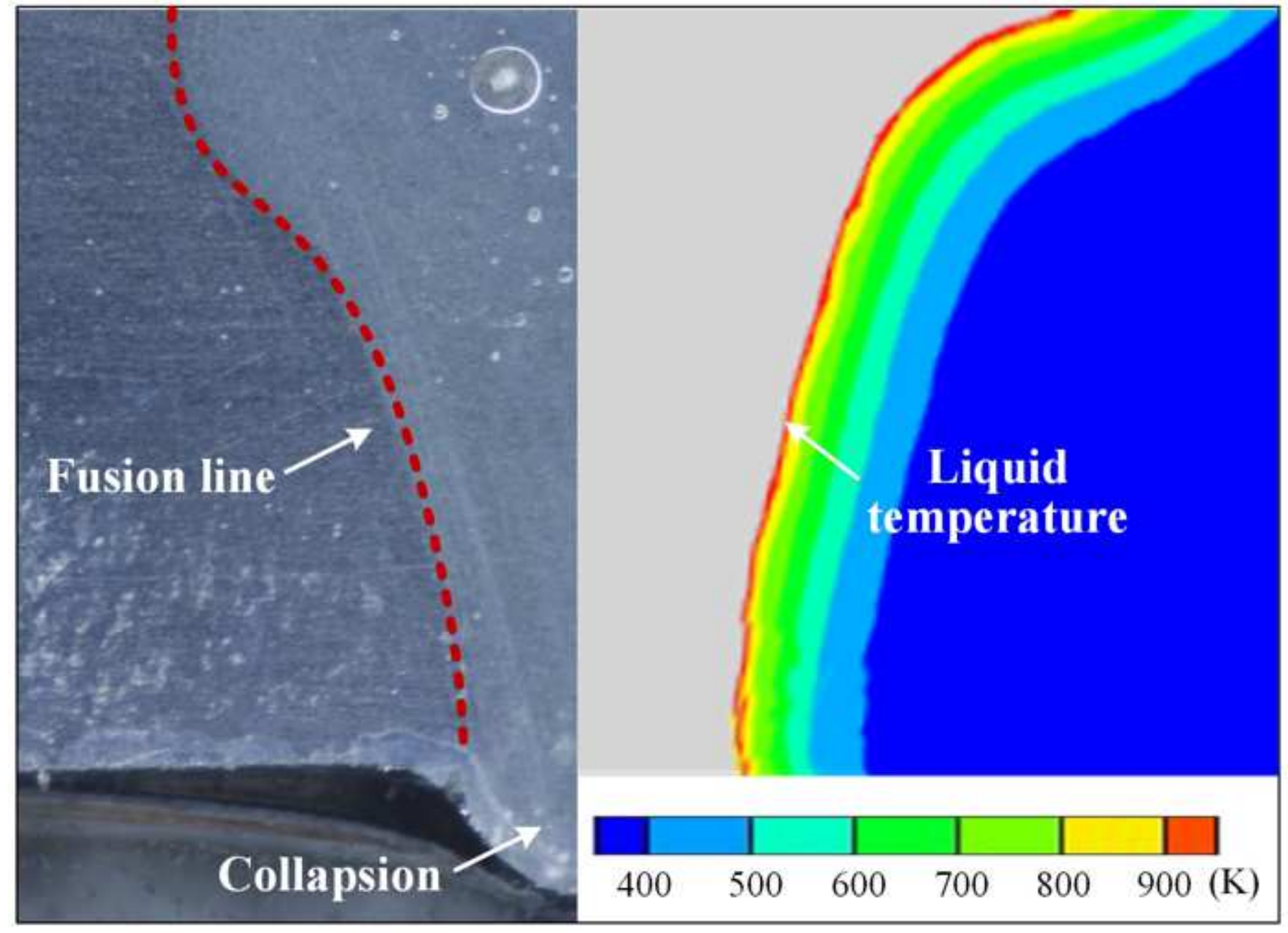

Figure 6

Comparison of the experimental result and simulation result 


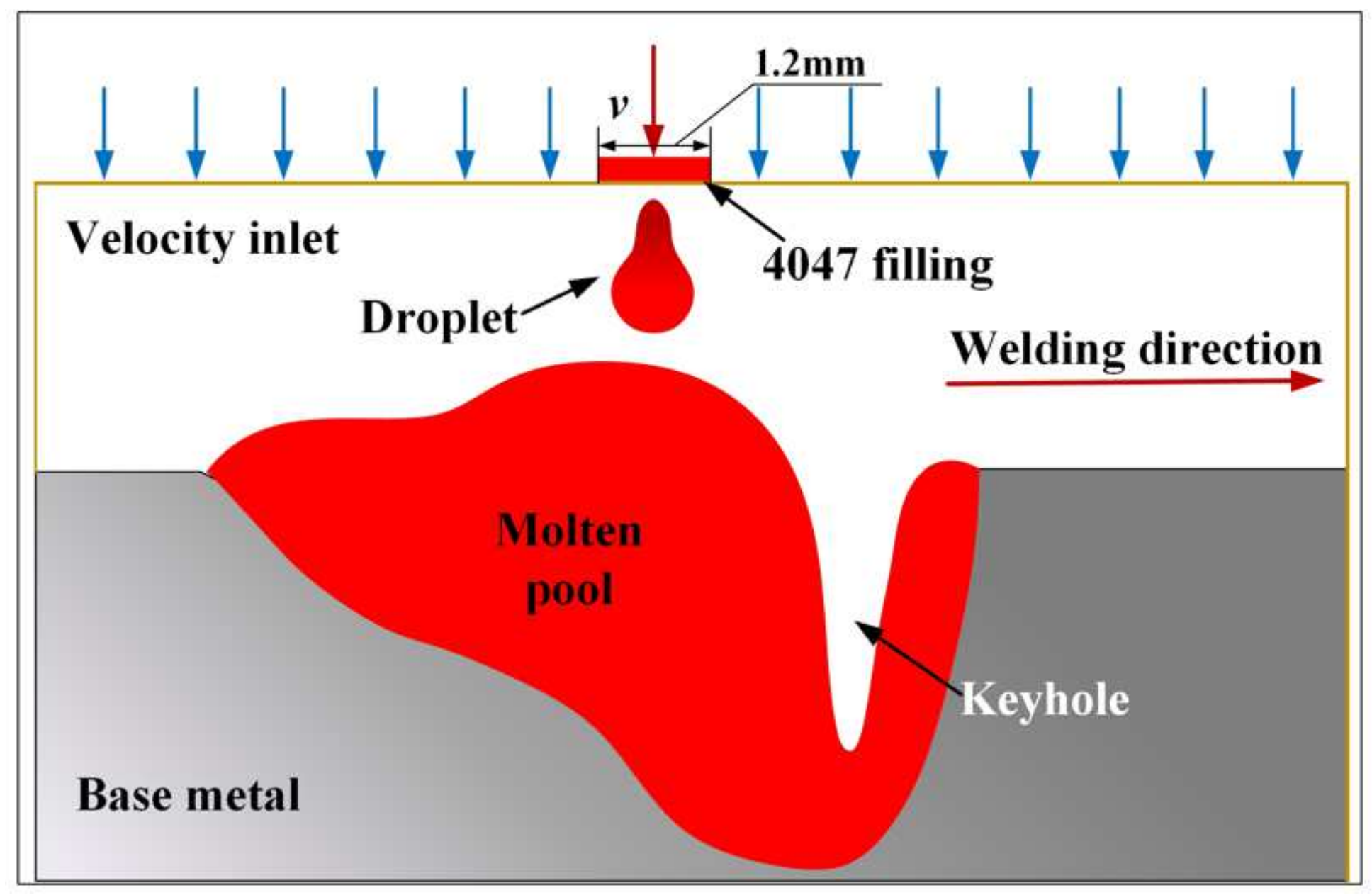

Figure 7

Geometric and boundary conditions of droplet transition model 

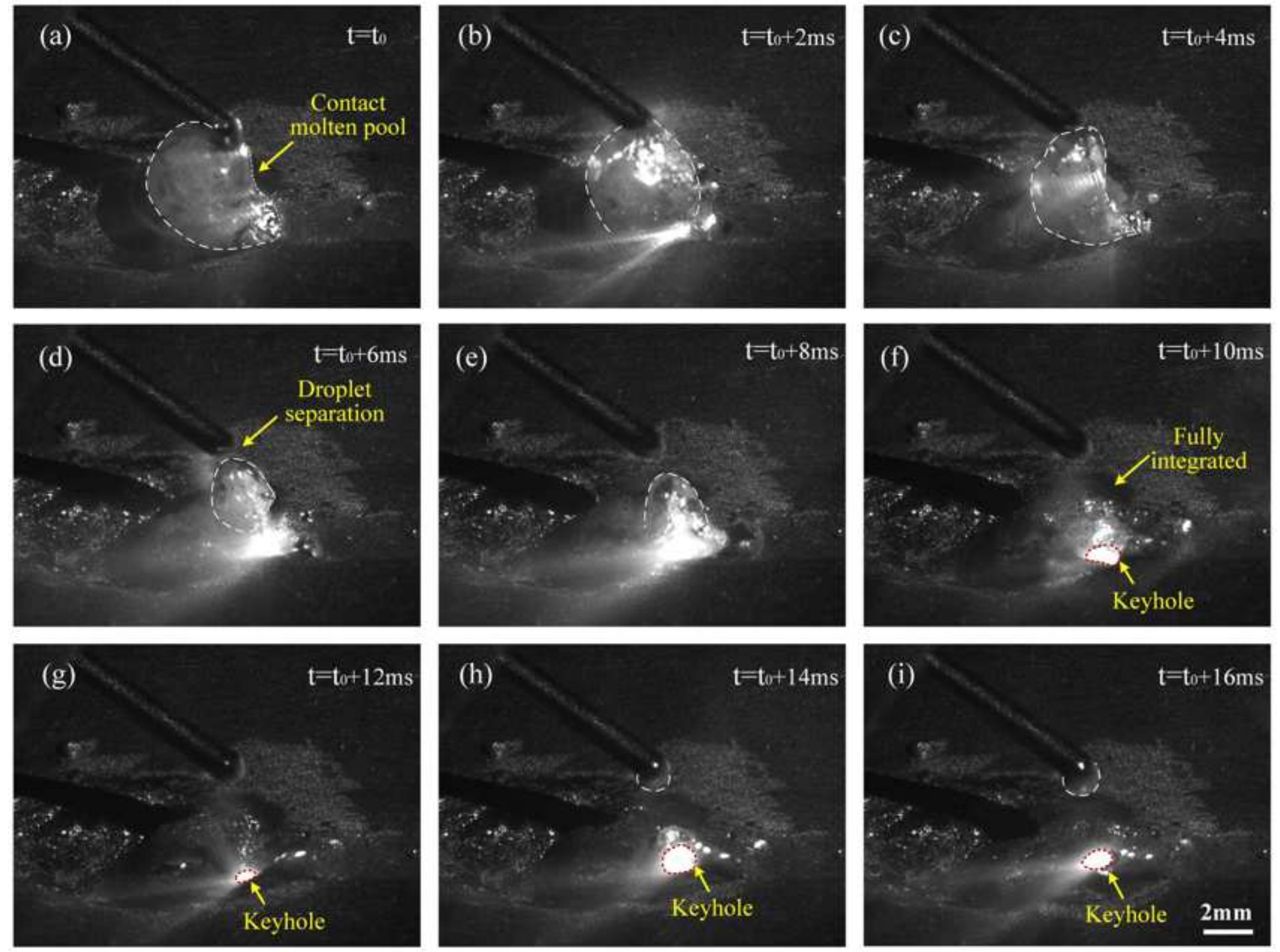

\section{Figure 8}

High-speed camera photos in the droplet transition process 


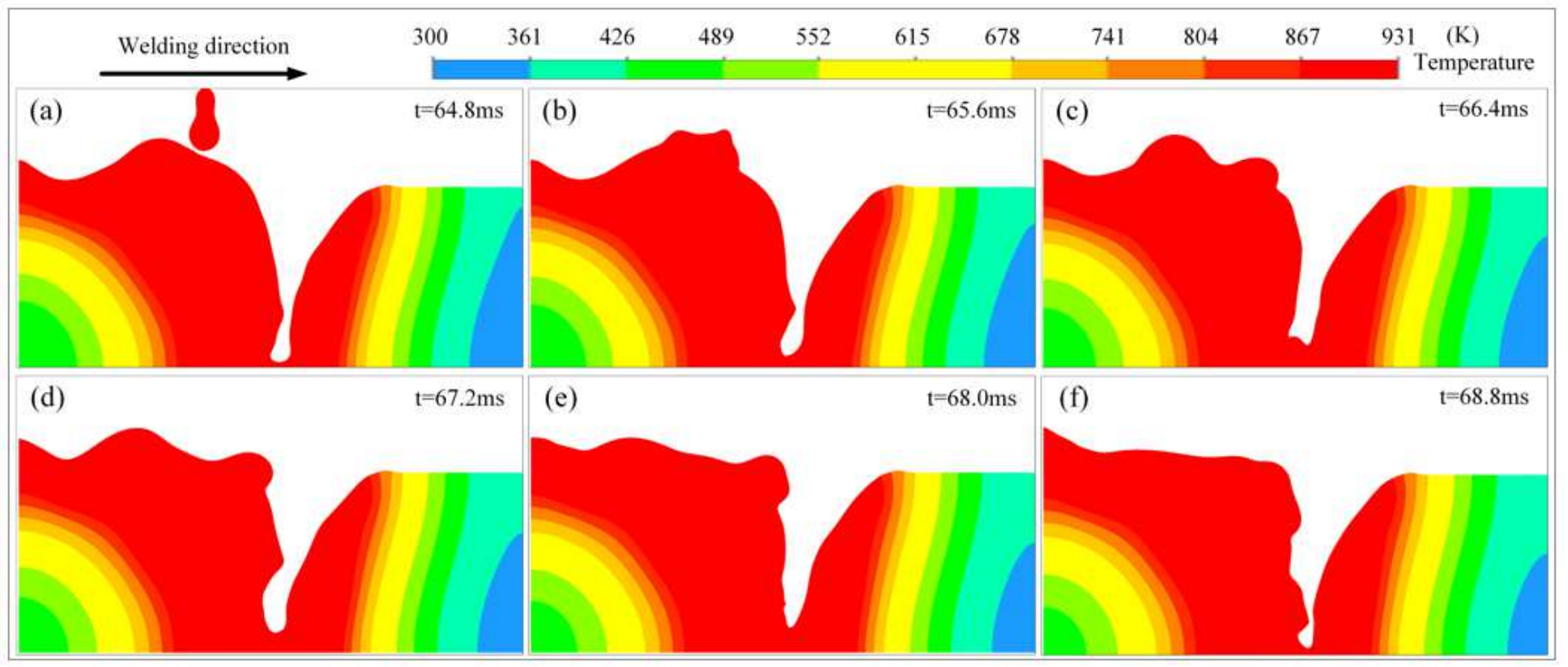

\section{Figure 9}

Distribution of temperature field in molten pool during droplet transition. (a) $t=64.8 \mathrm{~ms}$. (b) $t=65.5 \mathrm{~ms}$. (c) $t=66.4$ ms. (d) $t=67.2 \mathrm{~ms}$. (e) $t=68.0 \mathrm{~ms}$. (f) $t=68.8 \mathrm{~ms}$

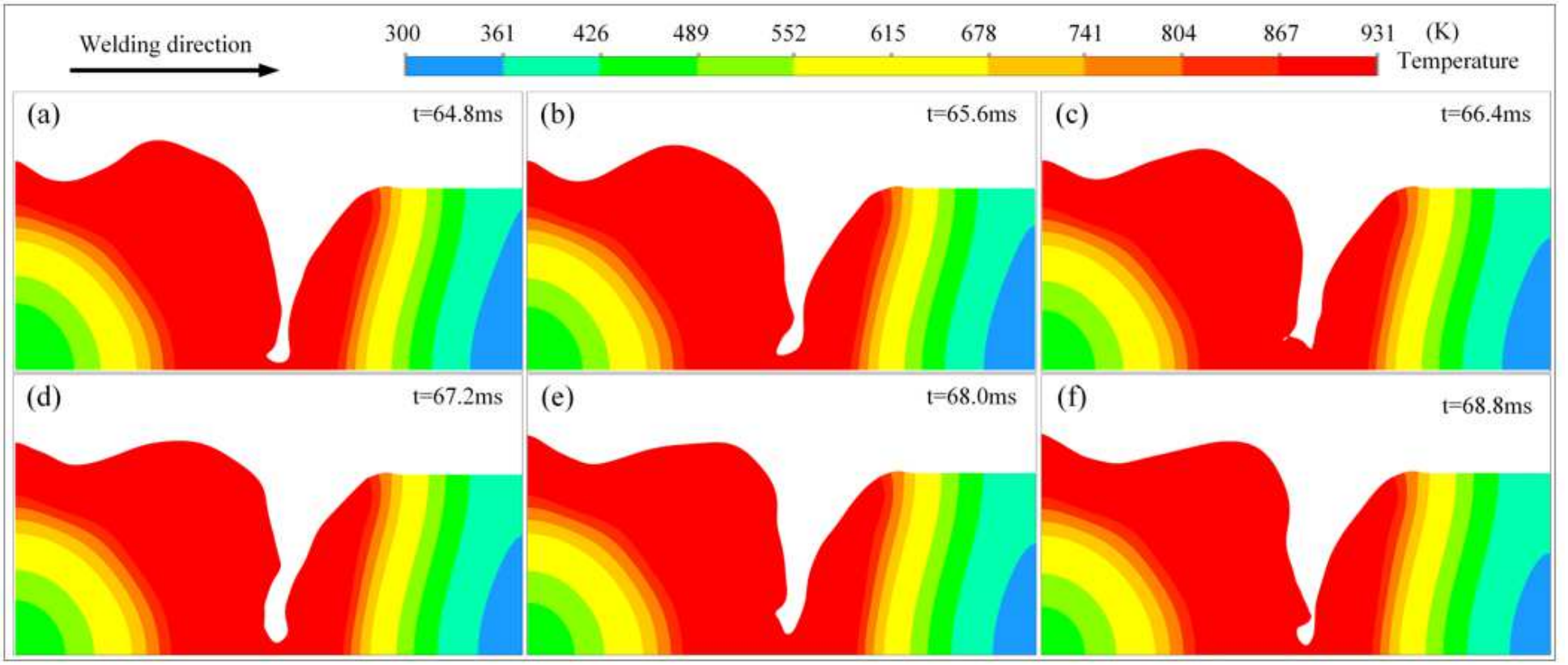

\section{Figure 10}

Distribution of temperature field in molten pool without droplet transition. (a) $t=64.8 \mathrm{~ms}$. (b) $t=65.5 \mathrm{~ms}$.

(c) $t=66.4$ ms. (d) $t=67.2 \mathrm{~ms}$. (e) $t=68.0 \mathrm{~ms}$. (f) $t=68.8 \mathrm{~ms}$ 


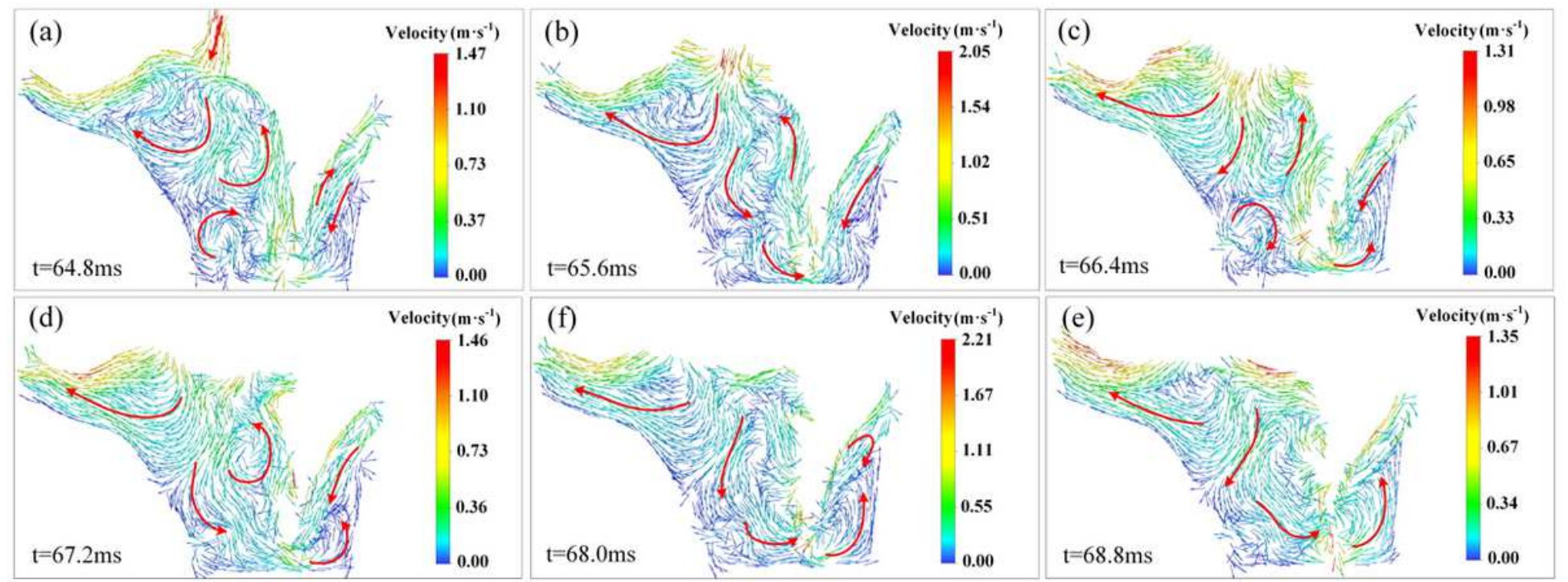

\section{Figure 11}

Distribution of flow field in molten pool during droplet transition. (a) $t=64.8 \mathrm{~ms}$. (b) $t=65.5 \mathrm{~ms}$. (c) $t=66.4$ ms. (d) $t=67.2$ ms. (e) $t=68.0$ ms. (f) $t=68.8 \mathrm{~ms}$

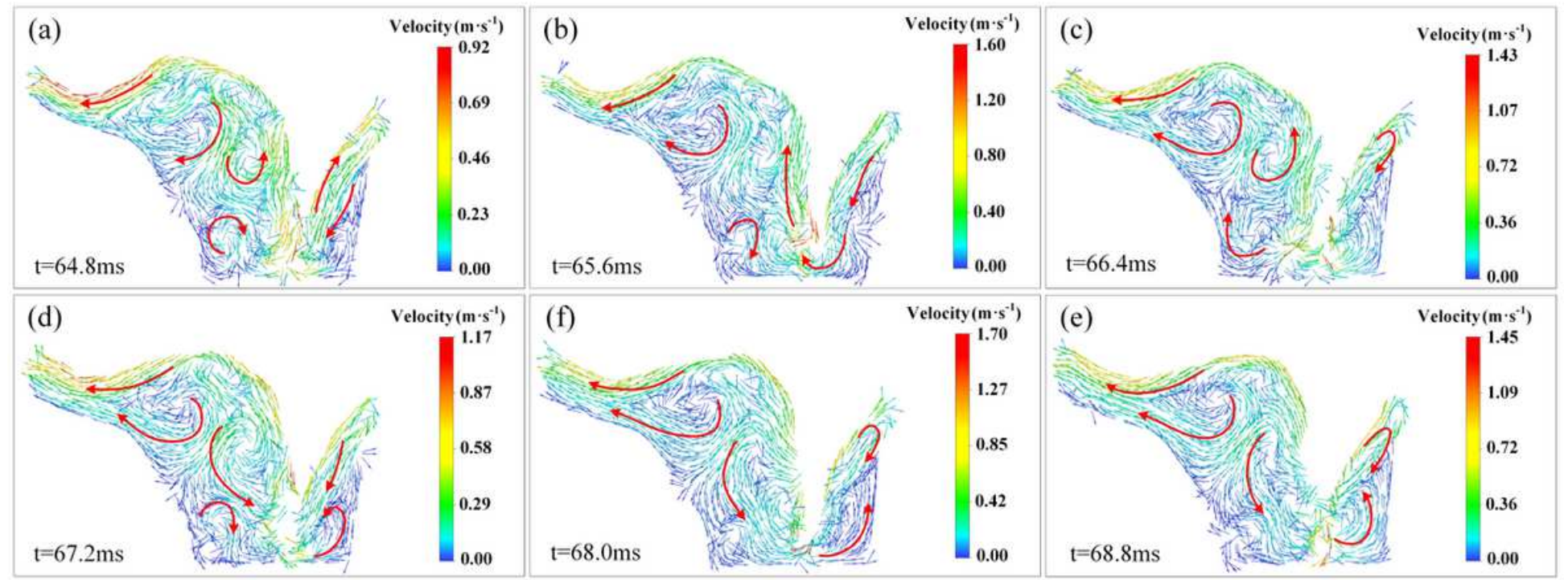

\section{Figure 12}

Distribution of flow field in molten pool without droplet transition. (a) $t=64.8 \mathrm{~ms}$. (b) $t=65.5 \mathrm{~ms}$. (c) $t=66.4$ ms. (d) $t=67.2$ ms. (e) $t=68.0$ ms. (f) $t=68.8 \mathrm{~ms}$ 

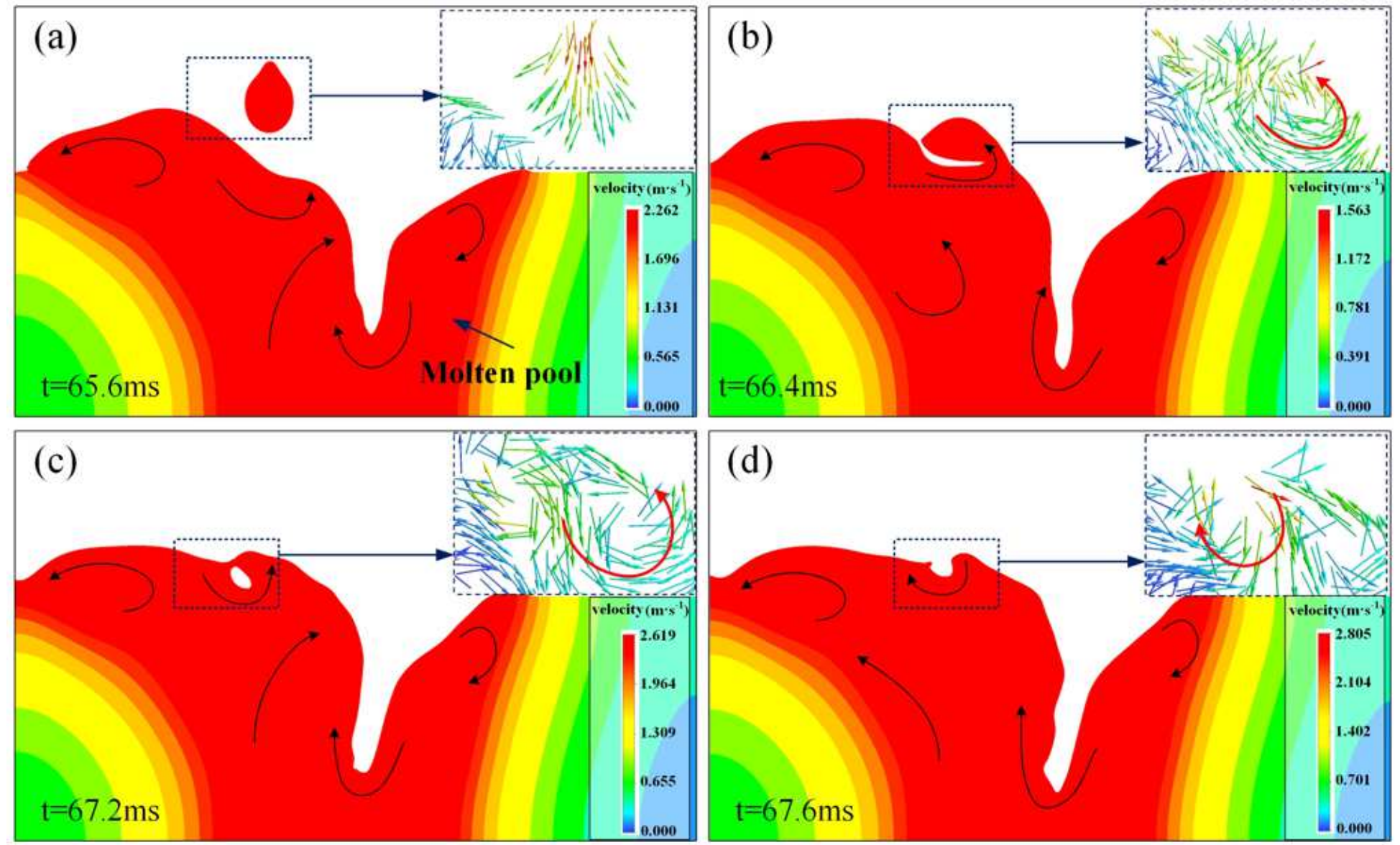

\section{Figure 13}

The trajectory of the bubble formed during the droplet transition. (a) $t=65.6 \mathrm{~ms}$. (b) $t=66.4 \mathrm{~ms}$. (c) $t=67.2$ ms. (d) $t=67.6 \mathrm{~ms}$
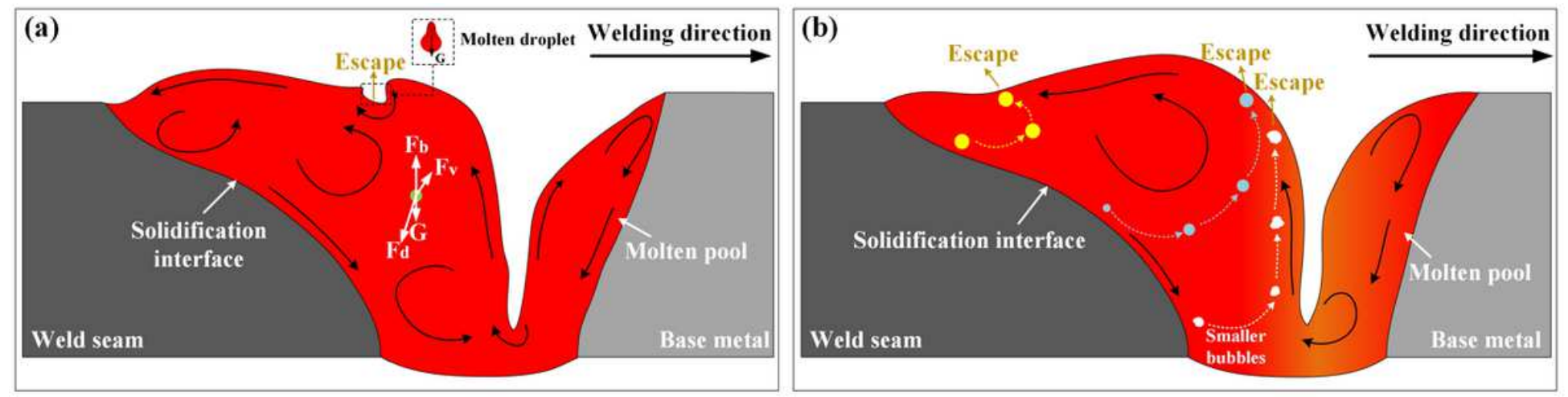

\section{Figure 14}

(a) The schematic diagram of the bubble trajectory during the droplet transition. (b) The schematic diagram of the bubbles trajectory in different positions of the molten pool 